This is a postprint version of the following published document:

Cavallaro, R., Demasi, L. Pasariello, A. (2014). Nonlinear Analysis of PrandtlPlane Joined Wings: Effects of Anisotropy. AIAA Journal, 52(5), 964-980

DOI: https://doi.org/10.2514/1.J052242

(C) 2014 American Institute of Aeronautics and Astronautics 


\title{
Nonlinear Analysis of PrandtlPlane Joined Wings: Effects of Anisotropy
}

\author{
Rauno Cavallaro, ${ }^{*}$ Luciano Demasi $†$ Andrea Passariello $\ddagger$
}

\begin{abstract}
Structural geometrical nonlinearities strongly affect the response of Joined Wings: it has been shown that buckling evaluations using linear methods are unreliable and only a fully nonlinear stability analysis can safely identify the unstable state. This work focuses on the understanding of the main physical mechanisms driving the wing system's response and the snap-buckling instability. Several counterintuitive effects typical of unconventional non-planar wing systems are discussed and explained. In particular, an appropriate design of the joint-to-wing connection may reduce the amount of bending moment transferred, and this is shown to eventually improve the stability properties although at price of a reduced stiffness. It is also demonstrated that the lower-to-upper-wing stiffness ratio and the torsional-bending coupling, due to both the geometrical layout and anisotropy of the composite laminates, present a major impact on the nonlinear response. The findings of this work could provide useful indications to develop effective aeroelastic reduced order models tailored for airplane configurations experiencing important geometric nonlinearities such as PrandtlPlane, Truss-braced and Strut-Braced wings, and Sensorcrafts.
\end{abstract}

\section{Introduction}

OINED Wings were proposed in the seventies ${ }^{1-3}$ for commercial transport and supersonic $\int_{\text {fighters. Joined Wings were also the subject of } U^{4,5} \text { and European }}^{6}$ patents. Many ad-

*PhD Candidate, Department of Aerospace Engineering, San Diego State University and Department of Structural Engineering, University of California San Diego, AIAA Member

${ }^{\dagger}$ Associate Professor, Department of Aerospace Engineering, San Diego State University, AIAA Member

${ }_{\ddagger}^{\ddagger}$ Visiting Graduate Student, San Diego State University, MS Candidate at the Dipartimento di Ingegneria Aerospaziale, Università di Pisa.

An earlier version of this paper (AIAA 2012-1462) was presented at the 53rd AIAA SDM conference, 23-26 April 2012, Honolulu, Hawaii, and was the recipient of the "Collier Research HyperSizer AIAA Structures Best Paper" award. 
vantages are claimed compared to classical cantilevered configurations: ${ }^{7,8}$ improved stiffness properties, high aerodynamic efficiency ${ }^{9}$ and superior stability and control characteristics. In addition to these theoretically significant advantages, a diamond Joined Wing can enclose a large antenna and be used for high altitude surveillance. ${ }^{10}$

For civil transportation, the PrandtlPlane has been analyzed in terms of aerodynamic performances, flight mechanics and controls, dynamic aeroelastic stability properties and preliminary design ${ }^{5,11}$

The design of Joined-Wing type of aircraft for civil transportation was also adopted in United States with the introduction of the concept of Strut-Braced Wings (SBW) ${ }^{12}$ and Truss Braced Wings (TBW). ${ }^{13}$

The growth of interest on Joined Wings led to both experimental ${ }^{14,15}$ and theoretical ${ }^{16,17}$ studies. These studies showed that the tools developed in decades and effectively used by the industry to analyze classical cantilevered wings need to take into account structural nonlinearities ${ }^{18,19}$ which are significant even for small angles of attack and attached flow. The significant forces and moments transferred through the joint make the geometric structural effects particularly important and linear aeroelastic models ${ }^{20}$ can give only a qualitative information on the instability properties but may miss important structural effects which should be taken into account. ${ }^{21,22}$ However, the adoption of fully-nonlinear structural models is impractical for design purposes especially if several alternative configurations are explored in an optimization ${ }^{20}$ effort. Ideally, the designer should have access to efficient reduced-order models. However, even well-established reduced order techniques ${ }^{23}$ based on second order modes $^{24,25}$ performed in an unsatisfactory manner when Joined Wings were considered. ${ }^{23,26}$

It was then realized that in order to effectively build a reduced order aeroelastic model specifically tailored for an efficient simulation with a full inclusion of the structural geometric effects, a physical understanding of the mechanism driving the nonlinear response of Joined Wings should be achieved. This is pursued in this work.

\section{Contributions of the Present Work}

PrandtlPlane configurations are joined-wing aircrafts designed for civil transportation. Thus, global snap-buckling instabilities are not acceptable. However, as discussed in reference [27] and in this work, a snap-buckling can take place even after a quasi-linear loaddisplacement response. This demonstrates the necessity of an understanding of the physics behind the instability phenomenon to avoid an abrupt change of state after a response which appeared to be linear.

For the PrandtlPlane-like configuration investigation was pursued in Reference [27]. The main results that were found could be summarized in the following main aspects. First, 
the strong nonlinear structural effects make the linear buckling analysis not very reliable as far as the static critical condition is concerned. Second, the system may be sensitive to snap-buckling type of instability under a certain combination of structural parameters. This led to the definition of the so-called Snap-Buckling Region which gives important indications on the design of these configurations. Third, it was shown that the load repartition between the upper and lower wings has a significant impact on the stability conditions: for a typical swept-back lower wing and swept-forward upper wing configuration more load on the upper wing alleviates the risk of instability. Fourth, some counterintuitive effects typical of this layout were discovered. For example, increasing the joint's size may be considered a not efficient design, since it could increase the height and this would appear unfavorable: it is well known that slender columns may increase the tendency to buckle. However, for aerodynamic-like mechanical loadings it was shown that the complex nonlinear response of the Joined Wing has actually an opposite effect and the stability properties are improved when the joint's height is increased. This has also practical implications since the induced drag is significantly reduced when the gap between the upper and lower wing is increased. Fifth, increasing the sweep angles was shown to dramatically reduce the snap-instability load.

These findings had relevant practical implications, but several questions needed an answer. In particular, the effects of composite materials required investigation since additional couplings could be introduced because of the anisotropy. Moreover, nowadays the adoption of composites is increasingly relevant (the new Boeing 787 and the Airbus 350 present a large percentage of structures designed with composites) and has to be considered also for Joined Wings. In addition, even for isotropic materials but general geometries (sweep angles, dihedrals, built-in twist), a realistic PrandtlPlane would present strong anisotropic behavior from a global point of view.

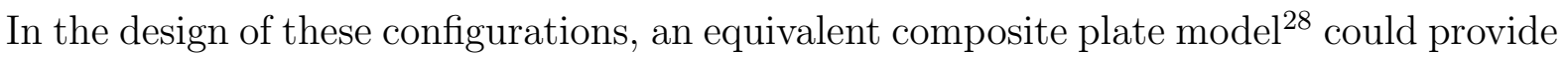
important indications. Thus, the present investigations based on plate-like models for the wings and the joint could also provide practical design information.

The JW models discussed and investigated in this work do not intend to reproduce the complex stiffness distributions of a realistic airplane configuration. The material properties and geometric dimensions are selected to be consistent with the ones typically adopted in wind tunnel models. Moreover, the materials are modified to explore how the different stiffness distributions affect the nonlinear response with particular emphasis on the snapbuckling instability.

This paper will provide contributions towards a fundamental understanding of the nonlinear response of PrandtlPlane Joined Wings. The first contribution concerns the role of the anisotropy (introduced by adopting composite materials) on the nonlinear response with 
particular emphasis on the snap-buckling instability. The second contribution is about the effects of the joint-wing connection (boundary conditions) on the geometrically nonlinear structural behavior. As suggested in Reference [29], since the system is statically indeterminate, significant reaction loads can buildup in the joint area that could have important consequences on stiffness and stability. Changing the wing-to-wing connection helps to isolate and better understand the phenomenon. The third contribution is finding the main driving mechanism which leads to the instability. It is shown that the bending moment transferring through the joint is determinant. Interesting features peculiar of the configurations which experience buckling are discussed with particular emphasis on the inward bending of the upper wing and rigid rotation of the joint in both chord-wise and span-wise directions. The fourth contribution is about the importance of the differential stiffness of the two wings: it will be shown that the stiffness ratio is one of the major parameters determining the instability risks. The upper wing (usually compressed under typical load conditions) needs to have a smaller relative stiffness. A surprising result with important implications.

The present work provides indications on the physical mechanisms of the nonlinear instability for PrandtlPlane configurations and Joined Wings. This could have practical application in the development of new and efficient aeroelastic reduced order models which could effectively adopt existing and reliable tools already in use in the aerospace industry but which cannot be directly extended for the Joined Wings without a proper understanding of the nonlinear phenomena.

\section{Nonlinear Structural Model}

The geometrically nonlinear finite element ${ }^{30}$ is based on the linear membrane constant strain triangle (CST) and the flat triangular plate element (DKT). The structural tangent matrix $\boldsymbol{K}_{T}$ is sum of two contributions: the elastic stiffness matrix, $\boldsymbol{K}_{E}$, and the geometrical stiffness matrix, $\boldsymbol{K}_{G}$.

The nonlinear governing equations are solved by adopting iterative methods such as Newton-Raphson and arc length techniques. ${ }^{31}$ After each iteration a displacement vector is obtained, rigid body motion is eliminated from elements and the pure elastic rotations and strains are found. ${ }^{30}$ Using these quantities the internal forces are updated for the next iteration.

The key relation which needs to be solved at each iteration ${ }^{27,32}$ involves the structural tangent matrix $\boldsymbol{K}_{T}^{\text {step } \mu \text { iter } n} \cdot \boldsymbol{u}^{\text {step } \mu \text { iter } n}$, the external non-follower loads $\boldsymbol{P}_{\text {ext }}$, and the array 
$\boldsymbol{P}_{\text {unb }}^{\text {step } \mu \text { iter } n}$ containing the unbalanced loads:

$$
\boldsymbol{K}_{T}^{\text {step } \mu \text { iter } n} \cdot \boldsymbol{u}^{\text {step } \mu \text { iter } n}=\underbrace{\left(\Lambda^{\text {step } \mu \text { iter }(n+1)}-\Lambda^{\text {step } \mu \text { iter } n}\right)}_{\lambda^{\text {step } \mu \text { iter } n}} \boldsymbol{P}_{\text {ext }}+\boldsymbol{P}_{\text {unb }}^{\text {step } \mu \text { iter } n}
$$

where the displacement $\boldsymbol{u}^{\text {step } \mu \text { iter } n}$ and, for the arc length case, the applied load fraction $\Lambda^{\text {step } \mu \text { iter }(n+1)}$ are unknown. Different closing constraint equations could be employed, leading to different arc length methods, such as Crisfield, Riks-Wempner or Ramm's (also called modified Riks) methods. ${ }^{31}$ As an example, application of Crisfield's cylindrical arc length method $^{31}$ leads to the following constraint:

$$
\left\|\boldsymbol{u}^{\text {step } \mu \text { iter } n}+\boldsymbol{U}^{\text {step } \mu \text { iter } n}-\boldsymbol{U}^{\text {step } \mu \text { iter } 1}\right\|^{2}=\Delta l^{2}
$$

where $\Delta l$ has been previously fixed. Equations 1 and 2 give raise to a second order relation for the $\lambda^{\text {step } \mu \text { iter } n}$.

It is worth to notice that the success of one of the arc length strategies in overcoming limit points is problem dependent. In some cases some strategies perform better than others, thus it may be necessary to switch between them to track the whole response curve.

The post-critical numerical analyses are inherently difficult to be carried out. It has been the authors' experience that a satisfactory performance of the finite element formulation in the pre-critical region does not imply a satisfactory performance on the post-critical region. Several numerical investigations showed that the terms of the out-of-plane contribution to the geometric stiffness matrix are crucially important on this regard.

Generally, Newton-Raphson procedures are preferred for computation of states far from limit points, for robustness and efficiency reasons. Moreover, to effectively track the curve beyond limit points it is necessary to adopt an arc length technique and restarting of the analysis from a converged state (restart capability), with the adoption of different set of parameters, may be necessary. This is the reason why an efficient technique which significantly reduces the needs of restarting analyses was implemented. In particular, the capability utilized in this work can automatically switch from Newton-Raphson to arc length strategy when close to a limit point. The opposite capacity to switch back to Newton-Raphson technique when far from limit points was also implemented. Furthermore, an automatic switch to different arc length techniques when the current one fails to overcome a limit point is also possible. More details are described in References [27,32]. 


\section{Description of the Analyzed Joined Wing Configurations}

Swept wings present a significant coupling between the bending and torsional deformations with important aeroelastic consequences. ${ }^{33}$ Due to the overconstrained nature of the Joined Wings, the sweep angle effects are even more determinant since the upper and lower wing are joined at the tip and the resulting structure is overconstrained. The bending-torsion coupling is more complex than a simple cantilevered wing and directly affects the stability properties and post-critical behavior of these configurations. Moreover, the composites can introduce some couplings which are not present in the case of isotropic materials, and with an accurate design the bending and torsional deformations may be modified to improve the overall response.

This work is mainly focused on the fundamental understanding of the geometric structural nonlinearity and the role it plays in the static instability for both unswept and swept Joined Wings. With this in mind, two configurations are discussed and analyzed. The first configuration is an unswept Joined Wing (Figure 1) and the second one (Figure 2) is a more realistic Joined Wing which presents a swept-back lower wing and a swept-forward upper wing. The loading condition is represented by a non-aerodynamic conservative vertical

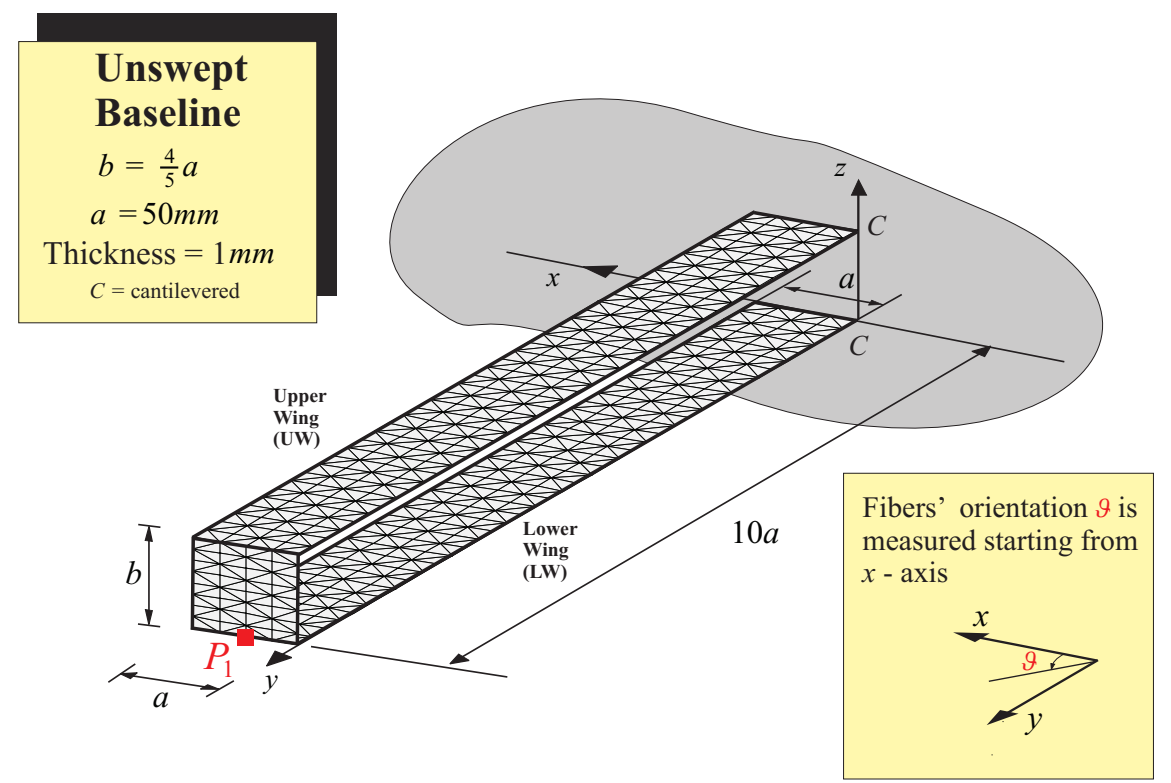

Figure 1. Unswept Baseline configuration, UREF.

pressure (direction $+z$ ) applied to both the upper and lower wings' surfaces (the joint is unloaded). The magnitude of the pressure is $p_{z}=0.55125\left[\frac{\mathrm{Kg}}{\mathrm{mm} \cdot \mathrm{s}^{2}}\right]$ and corresponds to a dynamic pressure relative to a speed of $V_{\infty}=30[\mathrm{~m} / \mathrm{s}]$. The thickness is held constant for both the wings and the joint and is equal to $1 \mathrm{~mm}$. Several materials will be adopted in this work to investigate the effects of composites on the nonlinear post-critical behavior of 


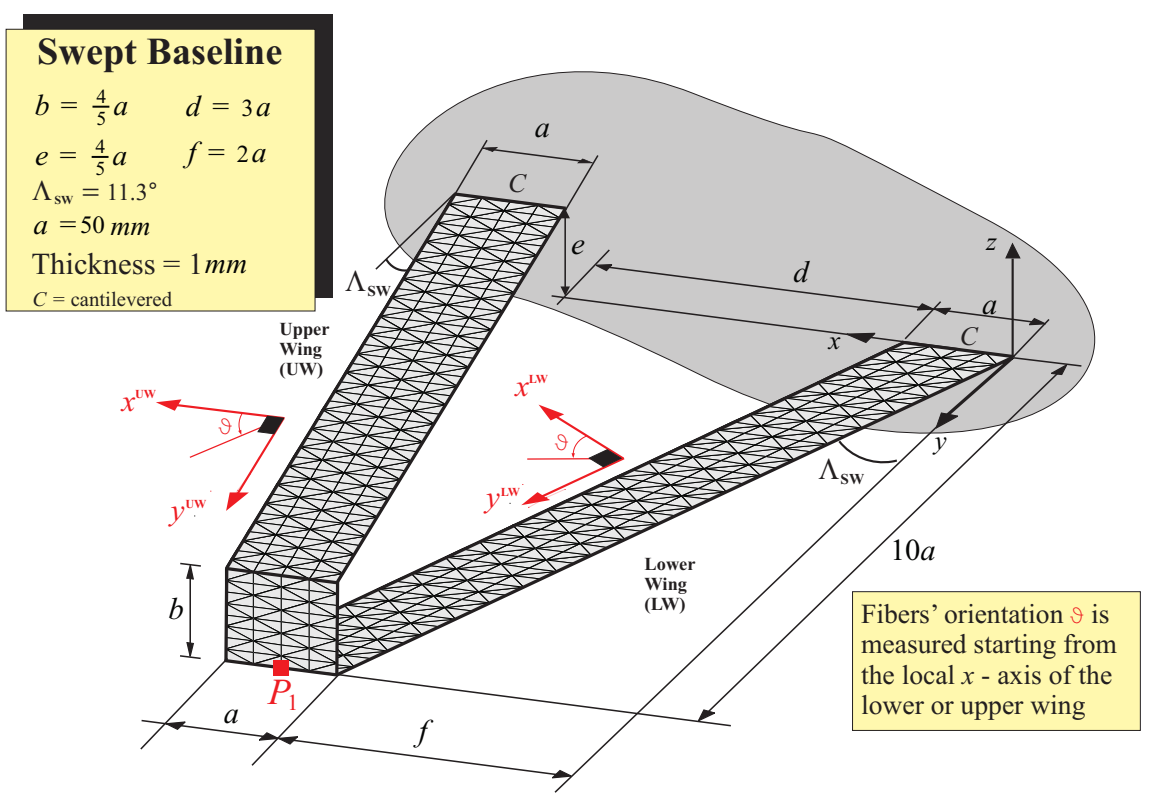

Figure 2. Swept Baseline configuration, SREF.

the Joined Wings. For a meaningful comparison of their effects, two Baseline configurations are defined for both the unswept and swept geometries reported in Figures 1 and 2. In particular, each baseline configuration presents a Young's modulus $E_{\mathrm{REF}}=6.9 \cdot 10^{7}\left[\frac{\mathrm{Kg}}{\mathrm{mm} \cdot \mathrm{s}^{2}}\right]$ and a Poisson's ratio $\nu_{\mathrm{REF}}=0.33$. The shear modulus is calculated from the well known relation $G_{\mathrm{REF}}=\frac{E_{\mathrm{REF}}}{2\left(1+\nu_{\mathrm{REF}}\right)}$. The baseline configurations are referred to as $U R E F$ and $S R E F$ for the unswept and swept cases respectively.

The results discussed in this work will present several investigations in which multilayer composite materials are adopted. For these cases the laminates' thicknesses are kept constant whereas the lamination schemes are changed. Each lamina or ply is identified by a material coordinate system which is in general not coincident with the global coordinate system adopted in the solution of the problem. For that reason it is necessary to specify the fibers' orientation angle at ply level. In this work the angle is measured starting from the wing's local $x$-axis: in the unswept case it coincides with the global $x$-axis, see Figure 1 , whereas for the swept case each wing has its own local reference $x$-axis $\left(x^{\mathrm{UW}}\right.$ and $x^{\mathrm{LW}}$ for the upper ad lower wings respectively: see Figure 2). The local $x$-axis is always perpendicular to the wing span direction and is not parallel to $x$ in the general case of swept Joined Wing.

A snapping phenomenon at global structural level (see also Reference [27]) as those that will be discussed here could not be accepted. It is also true that, when possible, the structures in aeronautical engineering are designed pursuing as much as possible a linear response. According to these observations, it may be stated, incorrectly, that a structural analysis may lose of interest well before a limit point is reached (see Reference [34] for a discussion about risks related to bi-stable regions). 
It may also be argued that the configurations for which snap occurs are subjected to a deformation which would not be realistic for a joined-wing aircraft.

However, the following observations could be made. First, the choice of the dimensions of the baseline models (see Figures 1 and 2) have been selected to be consistent with wind-tunnel scaled models. Second, the loads have been accordingly selected to observe the instability phenomenon, in an effort of conceptual understanding of the geometric nonlinearities and the effects of composite materials for both swept and unswept configurations. Third, HALE configurations typically undergo very large deformations, see for example Reference [35]. Lastly, it is very important for a thorough stability analysis the knowledge of different static equilibrium configurations at the same load level ${ }^{34}$.

The focus is on the understanding of the snap-buckling phenomenon ${ }^{27}$ and how the adoption of composite materials changes the strongly nonlinear structural behavior. Snapbuckling occurrence should be avoided. Composites provide a very effective option for the designer. How this can be practically achieved is extensively assessed in this work.

It should be pointed out that the efficient design of composite plate-like wings in view of achieving an optimal response (e.g., quasi-linear or snap-buckling-free response) has practical implications since a real wing-box structure could be eventually analyzed with an equivalent

plate representation. ${ }^{28}$ Thus, the analyses reported in this work could be adopted to gain directions about the design of a real snap-free joined-wing structure.

The main objective of this work is to shed some light on the physics related to the highly complex critical and post-critical behavior of composite anisotropic Joined Wing. Thus, the material properties used in the investigations are artificially modified to gain insights on the actual structural parameters which affect the structural response.

\section{Unswept Joined Wing Cases}

The unswept cases present the geometry shown in Figure 1 whereas the material properties are case-by-case changed to identify the important parameters affecting the nonlinear response. The joint transfers forces and moments between the wings. Thus, it is intuitive to expect a significant influence of the extensional and bending stiffness on the snap-buckling and post-critical responses. On this regard, if one considers the analogy with Euler's column and its instability properties when subjected to compressive forces, it could be inferred that when the two wings are loaded with a vertical pressure in the $+z$ direction the consequent compression of the upper wing is the driving mechanism to the instability. Thus, a design strategy aimed at increasing the extensional stiffness could be suggested. Actually, in this work a counterintuitive result will be demonstrated: the bending stiffness is the most relevant parameter which could not be easily predicted by simply using the joined-wing-analogue 
argument of Euler's column instability. Moreover, it will be shown that the bending stiffness ratio between the lower and upper wings is what regulates the snap-buckling for the unswept configuration reported in Figure 1.

\section{A. Lower-to-Upper Wing Stiffness Ratio and its Effects on the Snap-buckling}

The isotropic, orthotropic and anisotropic cases are now investigated.

\section{Isotropic case}

The Young's moduli of the upper and lower wing were varied to change the stiffness ratio, but in such a way as to maintain the linear response of point P1 of the UREF configuration, for details about the analytical expression see Appendix A of Reference [27]. In the process of varying the material of the wings (see Table 1), the joint's material has been held the same. All the analyses with the present software have been validated with NASTRAN, and the agreement is excellent. However, in many cases it was not possible to drive to convergence the commercial tool after the limit point: this is an indication of the numerical difficulties associated with this type of simulations for the case of Joined Wings and the necessity of the automatic switching features (from Newton-Raphson to arc length and vice-versa) implemented in the in-house capability. From Table 1 and Figures reported in Reference [32] and omitted here for brevity, it can also be observed that the lower-to-upper wing stiffness ratio $E^{r}=\frac{E^{\mathrm{LW}}}{E^{\mathrm{UW}}}$ plays an important role in determining the nonlinear response and snap phenomenon occurrence: increasing $E^{r}$ raises the snap load level (i.e., the first limit point encountered when tracking the response curve occurs at higher values of the load parameter $\Lambda$ ). Further increasing of the stiffness ratio $E^{r}$ postpones the buckling occurrence to higher level loads, and eventually it disappears and the response presents a stiffening effect (increasing of the load parameter/displacement slope). As Table 1 suggests, a critical value $E_{\mathrm{CR}}^{r}$ could be defined, which, for this particular case, is equal to 2.5 .

From the definition of $E^{r}$ it is deduced that increasing the stiffness of the lower wing compared to the stiffness of the upper wing is beneficial as far as the elimination of the snapbuckling is concerned. This is apparently a counterintuitive result since it would be expected that increasing the stiffness of the upper wing (the one which is compressed under this load condition) could be beneficial. It also confirms the fact that for Joined Wings the type of response does not follow the interpretation which could be used by adopting the classical arguments of the Eulerian compressed column. For an assigned load level, comparison of the deformed shapes corresponding to different values for the parameter $E^{r}$ showed $^{32}$ that the configurations on the verge of snapping (for that load level) present a deformation of the 


\begin{tabular}{|c|c|c|c|c|c|}
\hline \multicolumn{6}{|c|}{ Cases and relative parameters } \\
\hline Case ID & Wing & $\begin{array}{l}\text { Young's Modulus } \\
E \times 10^{-7}\left[\frac{\mathrm{Kg}}{\mathrm{mm} \cdot \mathrm{s}^{2}}\right]\end{array}$ & $\begin{array}{c}\text { Ratio } \\
E^{r}=\frac{E^{\mathrm{LW}}}{E^{\mathrm{UW}}}\end{array}$ & Snap & $\begin{array}{c}\text { Critical Load } \\
\Lambda^{\mathrm{CR}}\end{array}$ \\
\hline \multirow{2}{*}{ UREF } & Upper & 6.9 & \multirow{2}{*}{1} & \multirow{2}{*}{ YES } & \multirow{2}{*}{0.91} \\
\hline & Lower & 6.9 & & & \\
\hline \multirow{2}{*}{ UISO1 } & Upper & 12 & \multirow{2}{*}{0.2} & \multirow{2}{*}{ YES } & \multirow{2}{*}{0.81} \\
\hline & Lower & 2.4 & & & \\
\hline \multirow{2}{*}{ UISO2 } & Upper & 10 & \multirow{2}{*}{0.4} & \multirow{2}{*}{ YES } & \multirow{2}{*}{0.84} \\
\hline & Lower & 4.1 & & & \\
\hline \multirow{2}{*}{ UISO3 } & Upper & 8.7 & \multirow{2}{*}{0.6} & \multirow{2}{*}{ YES } & \multirow{2}{*}{0.86} \\
\hline & Lower & 5.2 & & & \\
\hline \multirow{2}{*}{ UISO4 } & Upper & 7.7 & \multirow{2}{*}{0.8} & \multirow{2}{*}{ YES } & \multirow{2}{*}{0.88} \\
\hline & Lower & 6.1 & & & \\
\hline \multirow{2}{*}{ UISO5 } & Upper & 6.0 & \multirow{2}{*}{1.3} & \multirow{2}{*}{ YES } & \multirow{2}{*}{0.95} \\
\hline & Lower & 7.8 & & & \\
\hline \multirow{2}{*}{ UISO6 } & Upper & 5.0 & \multirow{2}{*}{1.8} & \multirow{2}{*}{ YES } & \multirow{2}{*}{1.07} \\
\hline & Lower & 8.9 & & & \\
\hline \multirow{2}{*}{ UISO7 } & Upper & 4.5 & \multirow{2}{*}{2.1} & \multirow{2}{*}{ YES } & \multirow{2}{*}{1.22} \\
\hline & Lower & 9.5 & & & \\
\hline \multirow{2}{*}{ UISO8 } & Upper & 4.1 & 24 & YFS & 152 \\
\hline & Lower & 9.9 & & & \\
\hline UISO9 & Upper & 4.1 & 2.45 & YES & 1.68 \\
\hline & Lower & 10 & & & \\
\hline UISO10 & Upper & 4.0 & 25 & NO & $\infty$ \\
\hline & Lower & 10.1 & & & \\
\hline
\end{tabular}

Table 1. Details about the materials used for the different configurations. Poisson's ratio is $\nu=0.33$ for all cases.

upper wing characterized by a more pronounced inward bending deformation. This property is derived here for the isotropic case, but its validity is more general, as the discussion regarding the orthotropic materials will show.

Summarizing, to avoid snap-buckling and having on the contrary a stiffening effect, the ratio $E^{r}$ is one of the dominant parameters. In particular, a configuration featuring a value of this parameter larger than a critical value $E_{\mathrm{CR}}^{r}$, does not present a snap-buckling problem.

A stiffer lower wing (or alternatively a more compliant upper wing) is then desirable for avoiding the snap-buckling problem. In a real design the different stiffness of the two wings is likely to be connected with a difference share of the load carried by each wing. This also presents implications on the stress levels reached by the structure and has to be properly taken into account when these types of configurations are designed. 


\section{Orthotropic case}

It should be observed that an isotropic material does not present a preferential direction and, thus, the nature of the nonlinear response can be fully investigated only if anisotropic materials are adopted. As a first step towards this direction, the case of orthotropic plates is here analyzed.

The first test case involves a single lamina with fibers directed along the wing span. The fibers' angle $\vartheta$, measured counterclockwise from the $x$-axis is equal to 90 degrees, see Figure 3. This choice makes the material behavior to be orthotropic in respect of the free stream $x$ and span-wise $y$ directions. As done for the previously discussed isotropic case,

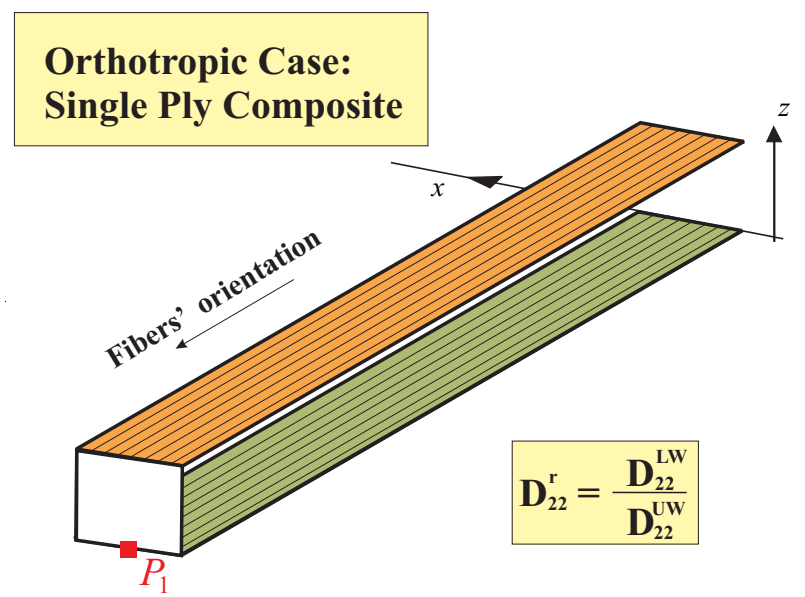

Figure 3. Unswept configurations for orthotropic cases and definition of lower-to-upper wing stiffness ratio.

the material properties are changed to numerically experiment the influence of the different parameters. However, modification of the properties is performed without changing point $P_{1}$ 's linear static response. This choice is useful for meaningful comparison of the different responses.

The material properties are selected as follows: $E_{2}^{\mathrm{LW}}=E_{2}^{\mathrm{UW}}=E_{\mathrm{REF}}, G_{12}^{\mathrm{LW}}=G_{12}^{\mathrm{UW}}=$ $G_{\mathrm{REF}}$, and $\nu_{12}^{\mathrm{LW}}=\nu_{12}^{\mathrm{UW}}=\nu_{\mathrm{REF}}$. The values of $E_{1}^{\mathrm{LW}}$ and $E_{1}^{\mathrm{UW}}$ are varied case by case.

The joint's material is fixed and is exactly the isotropic one used for the baseline case, $\operatorname{UREF}\left(E=6.9 \cdot 10^{7}\left[\mathrm{Kg} /\left(\mathrm{mm} \cdot \mathrm{s}^{2}\right)\right] ; \nu=0.33\right)$. Table 2 presents the analyzed orthotropic configurations. The parameter $E_{1}^{r}$ still affects the stability properties: if it is increased the snap buckling is eventually eliminated (UORTHO5). However, there are important quantitative differences. In fact as evident from isotropic configurations UISO10 response, with a value of $E^{r}=2.5 \equiv E_{\mathrm{CR}}^{r}$ buckling instability was avoided. On the contrary, even if the Joined Wing's geometry, the linear response of point $P_{1}$, and stiffness ratio are kept constant (with respect to UISO10), the orthotropic configuration UORTHO3 (featuring $\left.E_{1}^{r}>E_{\mathrm{CR}}^{r}\right)$ presents buckling as seen in Table 2. This suggests that, although $E_{1}^{r}$ is related 
to the physics of the phenomenon (increasing $E^{r}$ eventually eliminates the instability as seen in Table 2), from a quantitative point of view it is not the best choice to identify when the instability actually occurs. To find a more representative parameter linked to the stability

\begin{tabular}{|c|c|c|c|c|c|}
\hline \multicolumn{6}{|c|}{ Cases and relative parameters } \\
\hline Case ID & Wing & $\begin{array}{l}\text { Young's Modulus } \\
E_{1} \times 10^{-7}\left[\frac{\mathrm{Kg}}{\mathrm{mm} \cdot \mathrm{s}^{2}}\right]\end{array}$ & $\begin{array}{c}\text { Ratio } \\
E_{1}^{r}=\frac{E_{1}^{\mathrm{LW}}}{E_{1}^{\mathrm{UW}}}\end{array}$ & $\begin{array}{l}\text { Ratio } \\
A_{22}^{r}=D_{22}^{r}=\frac{D_{22}^{\mathrm{LW}}}{D_{22}^{\mathrm{UW}}}\end{array}$ & Snap \\
\hline \multirow{2}{*}{ UORTHO1 } & Upper & 5.0 & \multirow{2}{*}{1.8} & \multirow{2}{*}{1.7} & \multirow{2}{*}{ YES } \\
\hline & Lower & 8.9 & & & \\
\hline \multirow{2}{*}{ UORTHO2 } & Upper & 4.5 & \multirow{2}{*}{2.1} & \multirow{2}{*}{1.9} & \multirow{2}{*}{ YES } \\
\hline & Lower & 9.5 & & & \\
\hline \multirow{2}{*}{ UORTHO3 } & Upper & 4.0 & \multirow{2}{*}{2.5} & \multirow{2}{*}{2.2} & \multirow{2}{*}{ YES } \\
\hline & Lower & 10.1 & & & \\
\hline \multirow{2}{*}{ UORTHO4 } & Upper & 3.7 & \multirow{2}{*}{2.8} & \multirow{2}{*}{2.4} & \multirow{2}{*}{ YES } \\
\hline & Lower & 10.4 & & & \\
\hline \multirow{2}{*}{ UORTHO5 } & Upper & 3.4 & \multirow{2}{*}{3.2} & \multirow{2}{*}{2.7} & \multirow{2}{*}{$\mathrm{NO}$} \\
\hline & Lower & 10.8 & & & \\
\hline
\end{tabular}

Table 2. Details about the materials used for the different configurations. For each case it holds that $E_{2}=E_{\mathrm{REF}}, \nu=\nu_{\mathrm{REF}}, G=G_{\mathrm{REF}}$.

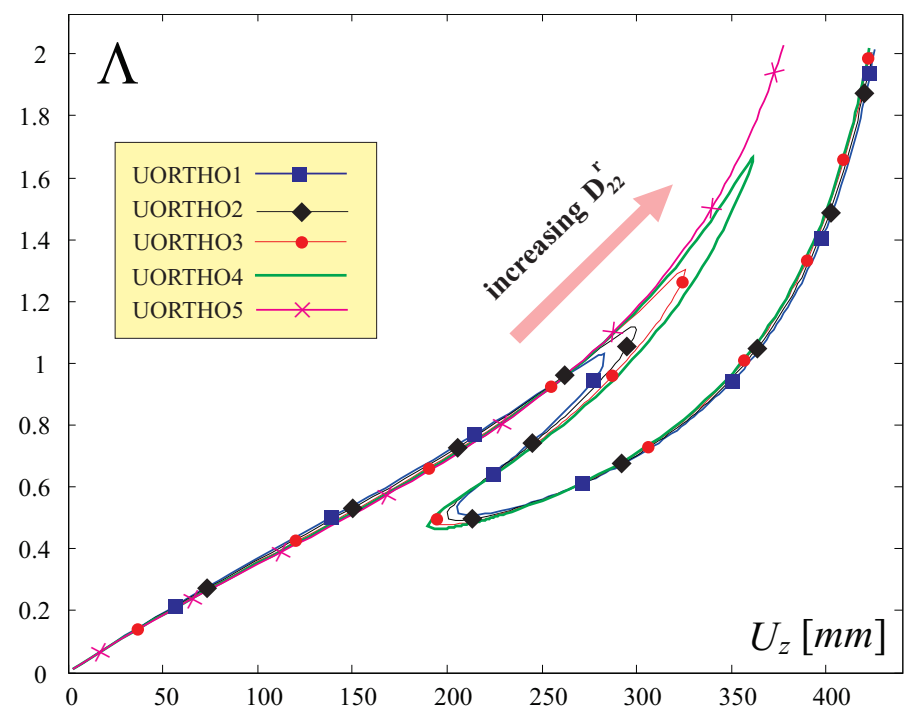

Figure 4. Load parameter $\Lambda$ versus cumulative vertical displacement $U_{z}$ of point $P_{1}$ for configurations employing different orthotropic wings. See Table 2 for details.

properties of the system and isolate the driving mechanism, the ratios of extensional and bending stiffnesses are then monitored. These ratios are indicated with the symbols $A_{m n}^{r}$ 
and $D_{m n}^{r}$ respectively. Their explicit definition is the following:

$$
A_{m n}^{r}=\frac{A_{m n}^{\mathrm{LW}}}{A_{m n}^{\mathrm{UW}}} \quad D_{m n}^{r}=\frac{D_{m n}^{\mathrm{LW}}}{D_{m n}^{\mathrm{UW}}}
$$

where $m$ and $n$ are indicies identifying each non-zero term of the corresponding matrix. The superscripts "LW" and "UW" indicates that the quantities are referred to the lower wing and upper wing respectively.

It is observed that each wing is modeled with a single lamina with constant thickness and material properties. This means that the matrix $\boldsymbol{A}^{r}$ which contains the ratios between the extensional stiffnesses is coincident with the matrix $\boldsymbol{D}^{r}$ which contains the ratios of the bending stiffnesses.

A series of investigations correlates the snap-buckling occurrence with the ratios $A_{22}^{r}$ and $D_{22}^{r}$ (see Table 2 and Figure 4). This is physically expected since $A_{22}^{r}$ relates the extensional stiffnesses in the wing span direction (important for example to describe the compression or tension of the wings) whereas $D_{22}^{r}$ relates the flexural stiffnesses (important in the determination of the principal bending moment of the wings). Moreover, the new critical parameter $\left[D_{22}^{r}\right]_{\mathrm{CR}}\left(\left[A_{22}^{r}\right]_{\mathrm{CR}}\right)$ has exactly the same value as the one for the isotropic case $E_{\mathrm{CR}}^{r}$, giving this a quantitative consistency.

Summarizing, it has been shown that the snap-buckling disappears when $A_{22}^{r}=D_{22}^{r}$ is larger than a critical value. Then the question is whether $A_{22}^{r}$ is the actual parameter that needs to be investigated/monitored or if $D_{22}^{r}$ is the one that needs to be considered or if both $A_{22}^{r}$ and $D_{22}^{r}$ are equally important. The answer represents a crucial concept in the design of a Joined Wing. For example, if $A_{22}^{r}$ is the most important term then the snap-buckling is mainly driven by compressive actions. On the other hand, if $D_{22}^{r}$ is the most important parameter then the snap-buckling occurs mainly because of bending actions. If the two parameters have similar relative importance, the physical mechanism is a combination of both compression and bending.

Since for a single orthotropic lamina it always is $A_{22}^{r}=D_{22}^{r}$, it is not possible to identify what is physically relevant as far as the instability is concerned unless a larger-than-one number of plies is selected so that it is possible to separately modify $\boldsymbol{A}^{r}$ and $\boldsymbol{D}^{r}$ matrices with the consequence that $\boldsymbol{A}^{r} \neq \boldsymbol{D}^{r}$.

In particular, several test cases have been introduced with the following assumptions: the lower wing is made of the same isotropic material employed for the reference case; the upper wing is made of a multilayered orthotropic composite laminate with layers made of the same material; the thickness of two generic different layers may be different, but the total thickness of the upper wing is maintained equal to $h=1 \mathrm{~mm}$. Table 3 shows all the analyzed cases and the values of $A_{22}^{r}$ and $D_{22}^{r}$ for each configuration. For these cases a reference closed-form 


\begin{tabular}{|l|c|c|c|c|c|}
\hline \multicolumn{2}{|c|}{ Cases and relative parameters } \\
\hline \multirow{2}{*}{ Case ID } & Wing & Lamination & $\begin{array}{c}\text { Ratio } \\
A_{22}^{r}=\frac{A_{22}^{\mathrm{LW}}}{A_{22}^{\mathrm{UW}}}\end{array}$ & $\begin{array}{c}\text { Ratio } \\
D_{22}^{r}=\frac{D_{22}^{\mathrm{LW}}}{D_{22}^{\mathrm{UW}}}\end{array}$ & SNAP \\
\hline UORTHOMP1 & Upper & $90_{0.15} / 0_{0.7} / 90_{0.15}$ & 2.5 & 1.3 & YES \\
\hline UORTHOMP2 & Upper & $90_{0.1} / 0_{0.35} / 90_{0.1} / 0_{0.35} / 90_{0.1}$ & 2.5 & 1.7 & YES \\
\hline UORTHOMP3 & Upper & $90_{0.05} / 0_{0.35} / 90_{0.2} / 0_{0.35} / 90_{0.05}$ & 2.5 & 2.7 & NO \\
\hline UORTHOMP4 & Upper & $90_{0.25} / 0_{0.5} / 90_{0.25}$ & 1.7 & 1.0 & YES \\
\hline UORTHOMP5 & Upper & $90_{0.1} / 0_{0.25} / 90_{0.3} / 0_{0.25} / 90_{0.1}$ & 1.7 & 1.7 & YES \\
\hline UORTHOMP6 & Upper & $90_{0.05} / 0_{0.25} / 90_{0.4} / 0_{0.25} / 90_{0.05}$ & 1.7 & 2.3 & YES \\
\hline UORTHOMP7 & Upper & $90_{0.03} / 0_{0.25} / 90_{0.44} / 0_{0.25} / 90_{0.03}$ & 1.7 & 2.9 & NO \\
\hline UORTHOMP8 & Upper & $90_{0.1} / 0_{0.8} / 90_{0.1}$ & 3.4 & 1.7 & YES \\
\hline UORTHOMP9 & Upper & $90_{0.07} / 0_{0.4} / 90_{0.06} / 0_{0.4} / 90_{0.07}$ & 3.4 & 2.2 & YES \\
\hline UORTHOMP10 & Upper & $90_{0.05} / 0_{0.4} / 90_{0.1} / 0_{0.4} / 90_{0.05}$ & 3.4 & 2.8 & NO \\
\hline
\end{tabular}

Table 3. Details about the materials used for the different configurations. For each case the lower wing is made of an isotropic material with $E^{\mathrm{LW}}=E_{\mathrm{REF}}, \nu^{\mathrm{LW}}=\nu_{\mathrm{REF}}$, where the upper wing features a composite material with plies laminated as indicate above. Each ply is manufactured with the same material $E_{1}=8.5 \cdot 10^{7}\left[\frac{\mathrm{Kg}}{\mathrm{mm} \cdot \mathrm{s}^{2}}\right], E_{2}=0.66 \cdot 10^{7}\left[\frac{\mathrm{Kg}}{\mathrm{mm} \cdot \mathrm{s}^{2}}\right], G_{12}=$ $0.56 \cdot 10^{7}\left[\frac{\mathrm{Kg}}{\mathrm{mm} \cdot \mathrm{s}^{2}}\right], \nu_{12}=0.28$.

analytical linear solution is impractical to obtain. Thus, it is not imposed to have the same slope (linear solution) for all the nonlinear responses relative to the cases reported in Table 3. However, this does not pose a conceptual limitation.

Figure 5 summarizes the responses of all the performed analyses. As reported in Table 3,
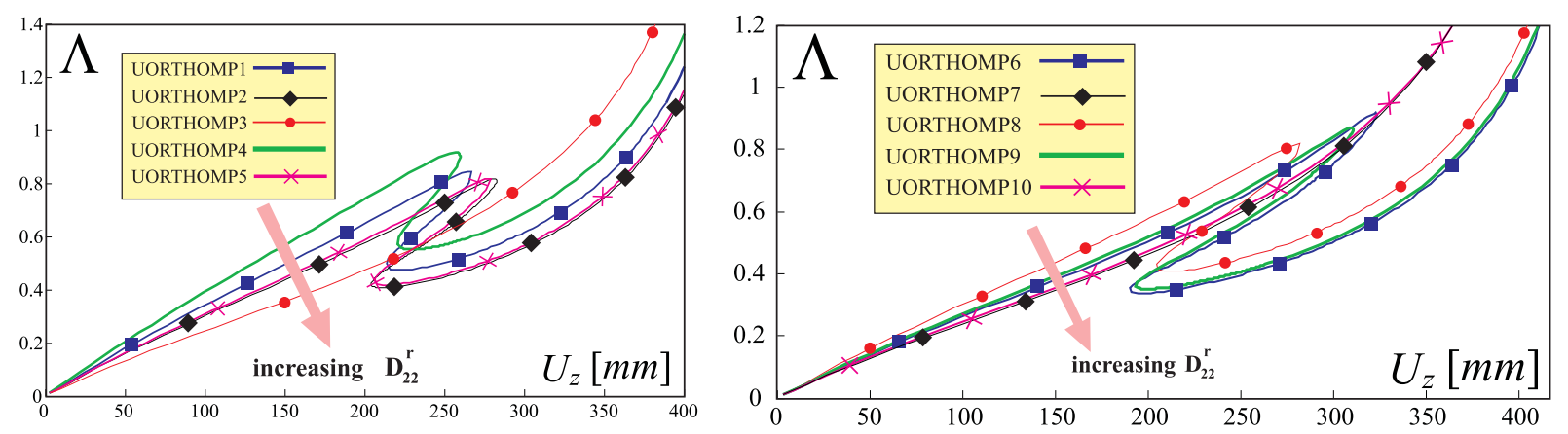

Figure 5. Load parameter $\Lambda$ versus cumulative vertical displacement $U_{z}$ of point $P_{1}$ for configurations featuring a lower wing composed of reference isotropic material $\left(E=E_{\mathbf{R E F}}\right.$ and $\left.\nu=\nu_{\mathrm{REF}}\right)$ and an upper wing composed of multi-ply composite material in order to have an orthotropic response. The different laminations are indicated in Table 3.

it is quite evident that $D_{22}^{r}$ plays the leading role, since it dictates snap occurrence, where this is not the case for the $A_{22}^{r}$ parameter. When $A_{22}^{r}$ is held constant and equal to 1.7 and $D_{22}^{r}$ is increased from 1.0 to 2.9, the snap-buckling disappears. Conversely, if $D_{22}^{r}$ is held 
constant and $A_{22}^{r}$ is varied, the response is not appreciably affected in terms of instability occurrence. Similar considerations, leading to the same conclusions, could be done for the other reported entries of $A_{22}^{r}$ and $D_{22}^{r}$.

The importance of the ratio $D_{22}^{r}$ is qualitatively consistent with Figure 6 which shows that when the snap-buckling occurs a more pronounced inward bending deformation of the upper wing is present compared to cases in which instability is not observed cases. More in detail, two configurations are depicted in Figure 6: one is associated with the case UORTHO1, which has $E_{1}^{r}=1.8$ and $D_{22}^{r}=A_{22}^{r}=1.7$, and the other one corresponds to the case UORTHO5, featuring $E_{1}^{r}=3.2$ and $D_{22}^{r}=A_{22}^{r}=2.7$. Configuration UORTHO1 incurs in a snap phenomenon and presents a larger inward bending for the upper wing.

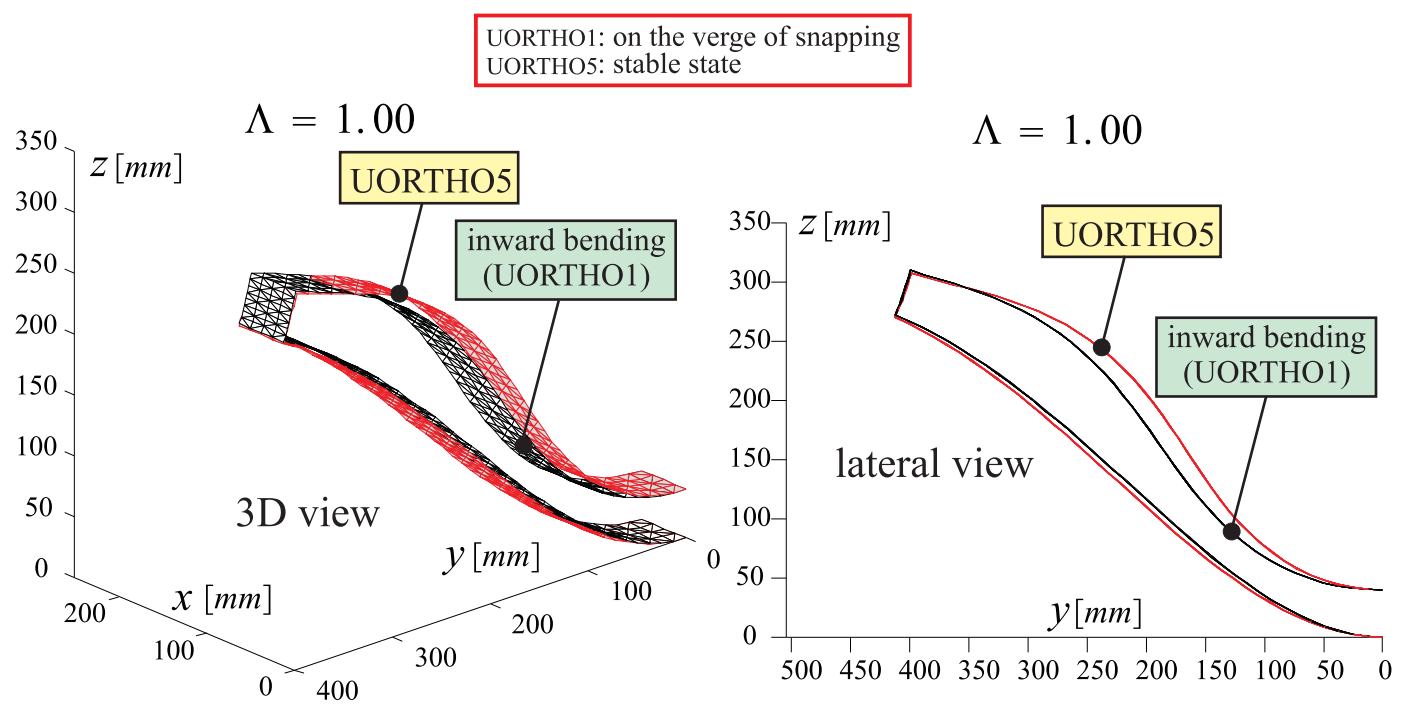

Figure 6. Comparison of configurations UORTHO1 and UORTHO5 at $\Lambda=1$.

It is interesting to observe that, both with and without the normalization (prescribed linear displacement of point $P_{1}$, adopted for the isotropic and single lamina orthotropic cases) the critical parameter $\left[D_{22}^{r}\right]_{\mathrm{CR}}$ keeps almost the same value (about 2.5).

It should be also observed that the isotropic case investigated in the preceding section and in Reference 32 can be seen as a particularization of the single-lamina orthotropic case investigated here. Thus, the physics ruling the snap buckling phenomenon is the same. However, in the isotropic case changing the extensional and bending stiffnesses independently from each other was not possible and so it was not possible to identify the bending stiffness ratio as the key parameter related to the stability properties of the system. 
3. Joint's Connection and Load Transferring Effects on the Snap-Buckling of Unswept Joined Wings

For both the cases of orthotropic and isotropic unswept Joined Wings, configurations which showed similar tip displacement for the same load level $\Lambda$ but different nonlinear behavior (i.e. one configuration experienced snap-buckling and the other did not, see for example Figure 6) were compared. One of the main features that was noticed was the different deformation of the upper wing. In particular, the inward bending of the upper wing was more pronounced for the model that was on the verge of snapping. It is true that the curvature distribution of the upper wing depends on all the transmitted force through the joint, being this exacerbated from the large displacement characteristic of the cases. However, it is of particular interest to monitor the bending moment $M_{y y}$ transmitted through the joint as a function of the load parameter $\Lambda$. More in detail, this is done for the configuration (see Table 1 ) which is on the verge of snapping (UISO 7 ) and for the configuration (presenting different material properties than the first one but with similar load-displacement curve up to that load level) which does not present buckling (UISO10).$^{32}$ Figure 7 shows the moment $M_{y y}$ on a finite element on the upper wing and near the joint. Although the interest is towards general behavior more

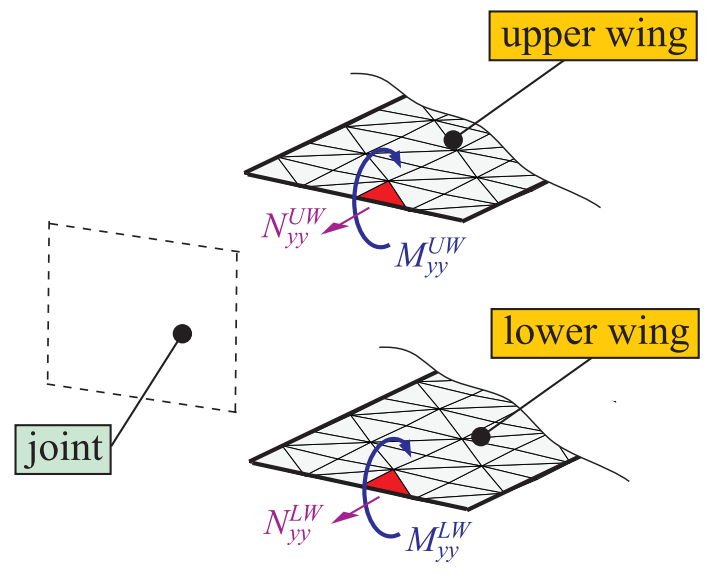

Figure 7. Wing span forces $\left(N_{y y}^{\mathrm{UW}}\right.$ and $\left.N_{y y}^{\mathrm{LW}}\right)$ per unit of length and primary bending moments $\left(M_{y y}^{\mathrm{UW}}\right.$ and $M_{y y}^{\mathrm{LW}}$ ) per unit of length transferred to the upper and lower wings. The forces and moments are calculated at the centroid of the finite elements shown in the figure.

than specific values, to have more reliable predictions a refined model using approximately 15000 DOF (against the approximately 2600 DOF of the base model), is used for comparison purposes. These models show an almost perfect agreement in terms of cumulative vertical displacement of point $P_{1}$, see Figure 8 . As it is well known, the force and moments (see Figures 9 and 10) converge more slowly when the mesh is refined. The correlation of their trends is very good. It should also be pointed out that since the forces and moments per unit of length are evaluated at the centroid of of the elements (see Figure 7 for the base model) a refining of the mesh implies a calculation of these quantities on a different (but 

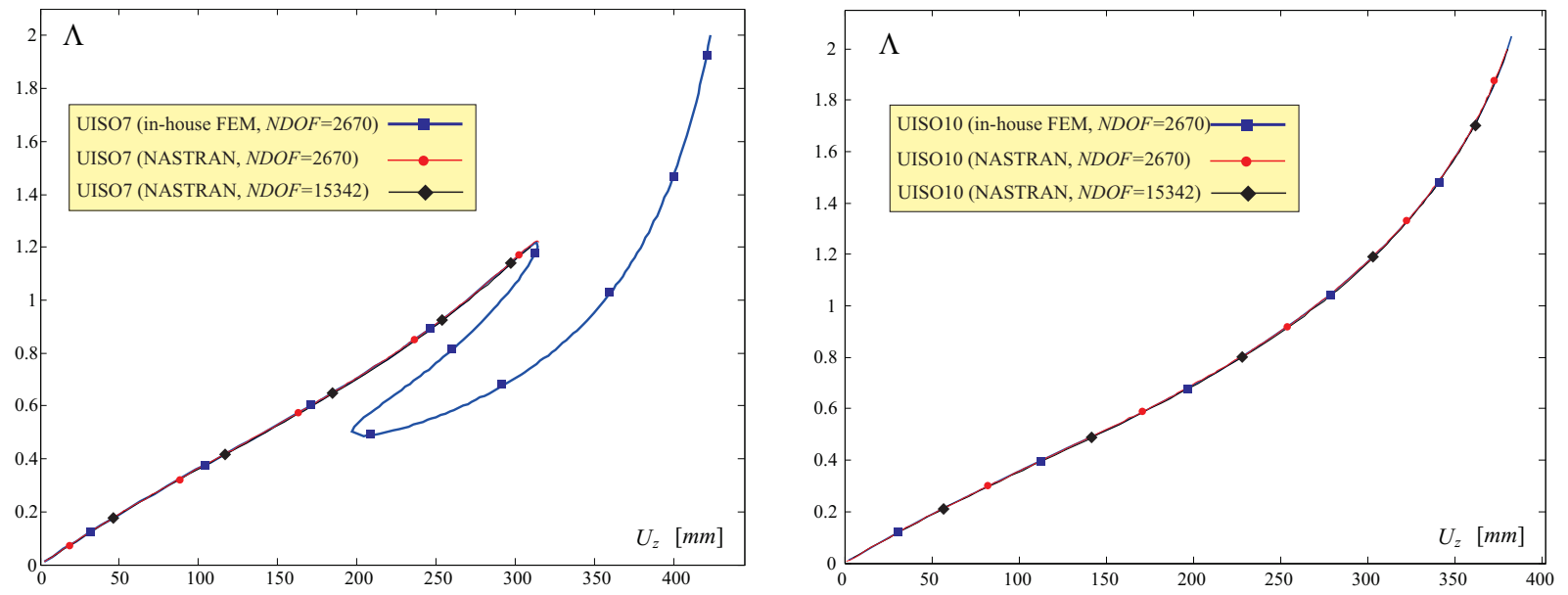

Figure 8. Curves of the load parameter $\Lambda$ versus cumulative vertical displacement $U_{z}$ of point $P_{1}$ for UISO7 (on the left) and UISO1O (on the right) obtained from simulations using different FE solvers and mesh sizes. Both NASTRAN solutions showed convergence problems after the first limit point.

close) point. The interest of this discussion is to show the trends. Thus, this fact does not affect the following discussion.

In Figure 9 the value of $M_{y y}$ is plotted for both the upper and lower wings for both the cases. Considering the upper wing, it is possible to observe that $M_{y y}^{\mathrm{UW}}$ shows similar trend in the pre-buckling area. However, the configuration which does not experience snapbuckling (UISO10) presents a larger moment $M_{y y}^{\mathrm{UW}}$ compared to the one corresponding to UISO\%. At a certain load parameter, smaller than the critical value, the moment relative to configuration UISO'7 starts diminishing in value and eventually the snap-buckling occurs. For the lower wing the bending moments of the UISO'7 and UISO10 configurations are practically identical. For completeness, Figure 10 shows the force per unit of length $N_{y y}$ on the upper wing (see also Figure 7).

The different trends regarding the transmitted bending moment (see Figure 9 a)) suggest that the snap-buckling occurrence could be strictly tied with $M_{y y}$. To further demonstrate this observation, the boundary conditions between the joint and the upper (or lower) wings are now modified to reduce the amount of moment which is transferred. This is accomplished by the adoption of a multifreedom constraint which allows the joint-upper-wing or joint-lower-wing relative rotation. To simulate some stiffness of the joint a relatively small torsional spring $\left(k_{\vartheta}=100 \frac{\mathrm{Kg} \cdot \mathrm{mm}^{2}}{\mathrm{~s}^{2} \cdot \mathrm{rad}}\right)$ has also been added at the joint-wing connection. It should be noted that a large value for the spring stiffness would correspond to a perfect joint's connection of the types analyzed so far, whereas a zero-value for the stiffness of the spring would correspond to a perfect hinge connection. Since the adopted value for the torsional stiffness is quite small compared to the stiffness of the finite elements, the simulated joint-wing connection is similar (but not equivalent) to a hinge connection. This set of 
a)

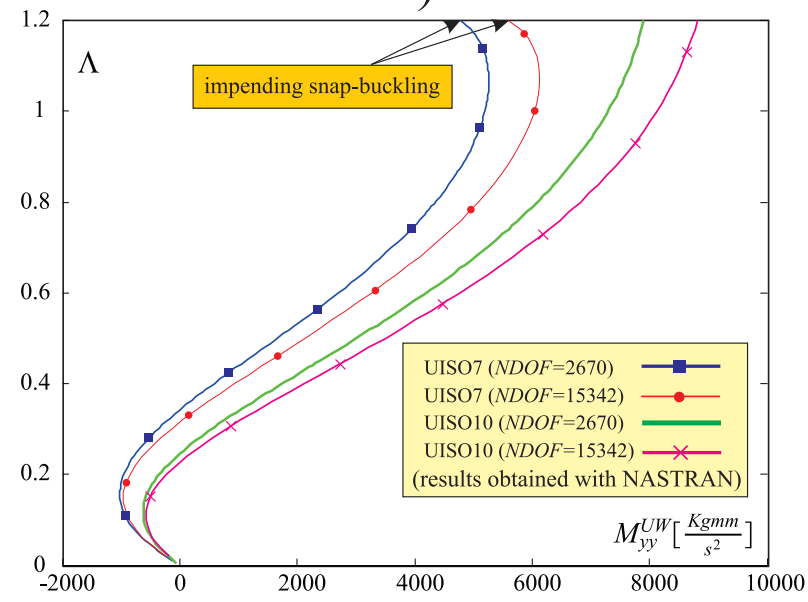

b)

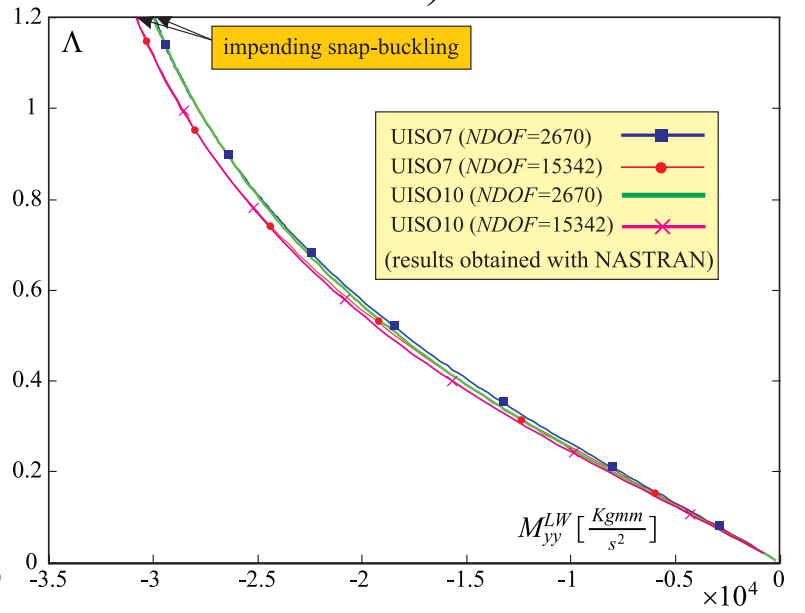

Figure 9. Bending moment per unit of length $M_{y y}$ on the upper wing, a), and lower wing, b). UISO7 and UISO1O cases. See also Figure 7 for a graphical representation.

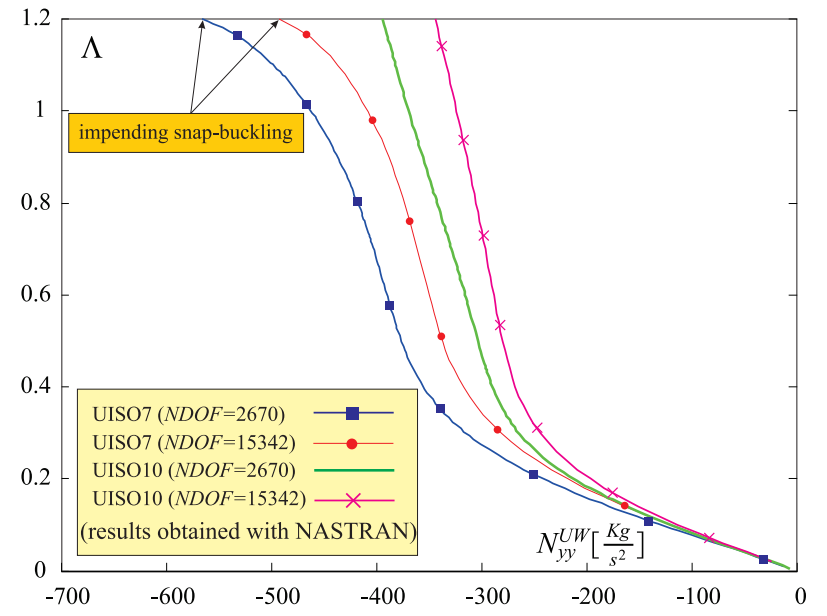

Figure 10. In-plane force per unit of length $N_{y y}$ on the upper wing for the UISO7 and UISO1O cases. See also Figure 7 for a graphical representation.

boundary conditions is referred as quasi-hinge connection in this work.

A quasi-hinge connection reduces the amount of moment transferred by the joint to the wing. Thus, it is expected that this connection has the tendency to reduce or eliminate the buckling occurrence. To prove that, three configurations are based on UREF are considered: one with perfect joints, one with a quasi-hinge connection between the joint and upper wing, one with a quasi-hinge connection between the joint and the lower wing. The related nonlinear responses are plotted in Figure 11. It can be observed that the snap-buckling disappears in both cases in which a quasi-hinge connection is employed. Moreover, reducing the bending moment transmission prevents the snap to occur. Moreover, if the responses relative to these cases are superimposed, it is possible to realize that the pre-critical states for the perfect joint case show a larger value of the stiffness (i.e., higher slope of the displacement-load 


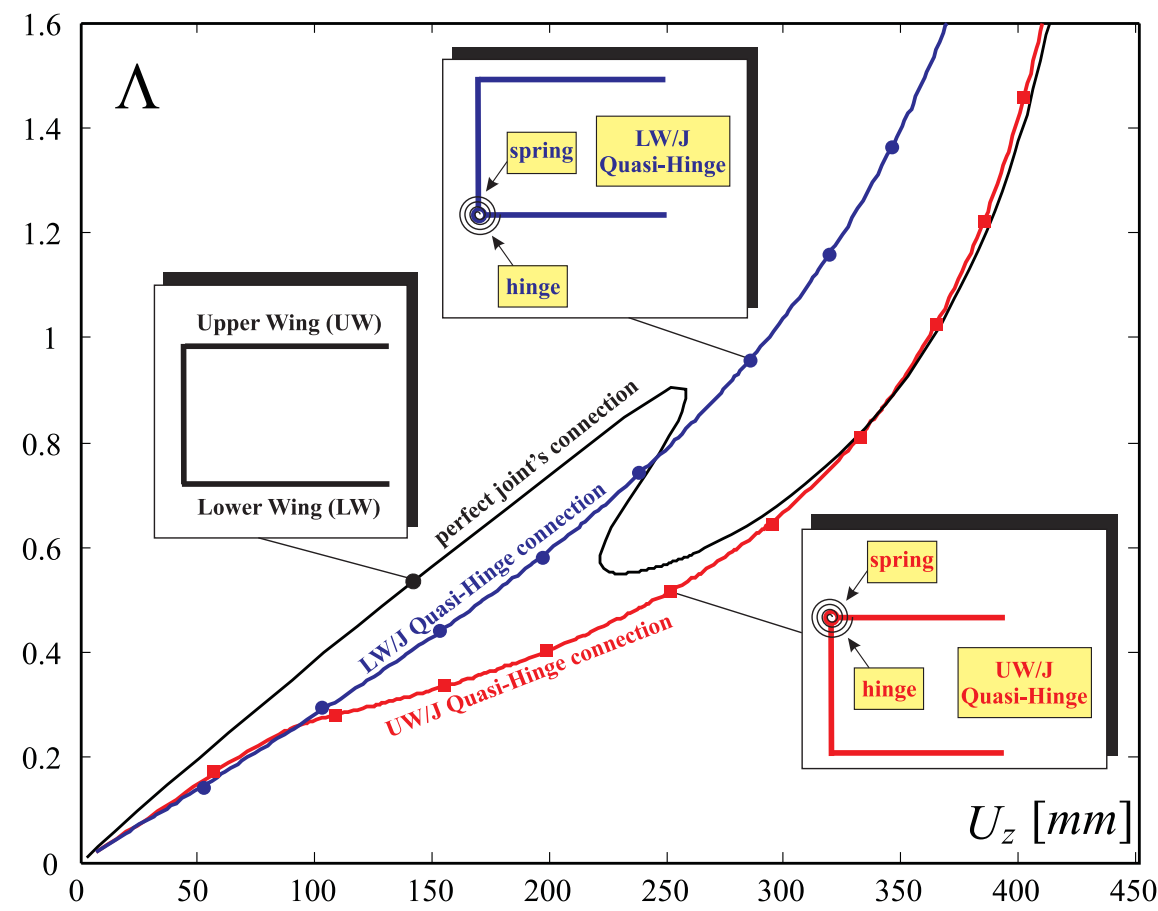

Figure 11. Cumulative vertical displacement $\left(U_{z}\right)$ of point $P_{1}$ versus load factor $(\Lambda)$ for $\boldsymbol{U R E F}$ configurations when a rigid or quasi-hinge connection is used between the joint and the upper or lower wing. $\mathrm{UW} / \mathrm{J}$ and $\mathrm{LW} / \mathrm{J}$ are abbreviations for indicating a quasi-hinge connection between upper wing and joint or between lower wing and joint respectively.

curve in Figure 11). Thus, the configurations featuring a quasi-hinge connection experience a reduction of stiffness, which is more pronounced when the quasi-hinge connection is located between the joint and the upper wing. Summarizing, the presented analyses lead to
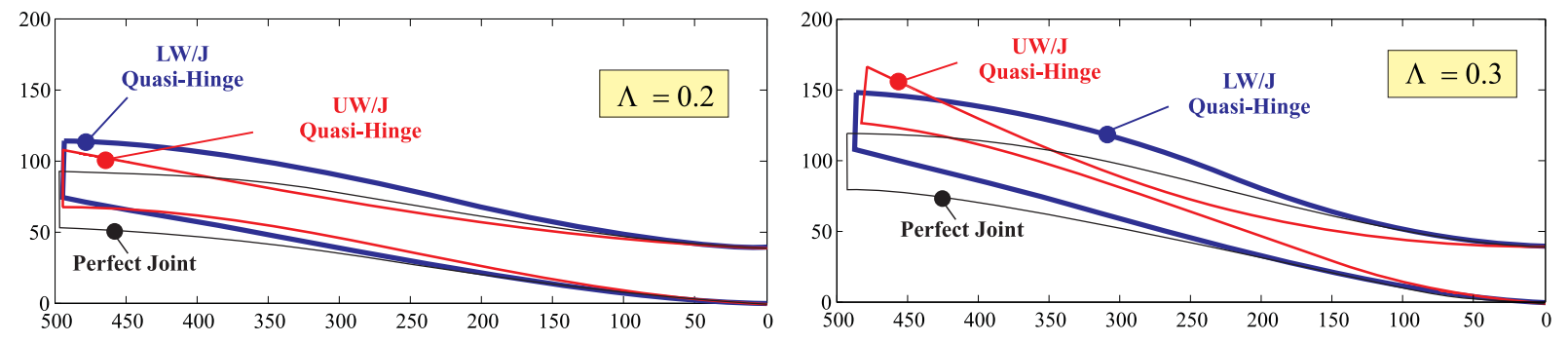

Figure 12. Comparison of the deformed structures for different $(\Lambda)$ values, for the perfect and quasi-hinge connection cases. $\mathrm{UW} / \mathrm{J}$ and $\mathrm{LW} / \mathrm{J}$ are abbreviations for indicating a quasi-hinge connection between upper wing and joint or between lower wing and joint respectively.

the conclusion that bending action transmission is one of the main sources of non-linearities when stability is concerned. When the perfect joint is considered the stiffness of the system is first increased but eventually the instability phenomena occurs (see Figure 11). When the bending moment is partially transmitted, the response does not present any snap-buckling phenomenon. However, there is a consistent loss of stiffness (see Figure 11). 
Studies on the connection between wings have already been undertaken for example by Stearman, Lin, and Jhou (Reference [29]). In that work, the rigid connection was assessed as being the most favorable in terms of root bending moment alleviation and stiffness. And, at least from a stiffness perspective, the results are in agreement with the results presented in the current work. However, the analyses of Reference [29] were obtained with linear models, and thus, phenomena such as the snap-buckling could not have been predicted or included. Furthermore, it should be noted that the conclusions applies for the particular configuration (a sensorcraft/joined-wing one) and load condition. The highly complex structural response of the Joined Wings need careful investigations case by case.

The authors also like to quote a passage from Reference [29] in which it is stated that the overconstrained nature of the system could give rise to significant load transfer through the joint which could be detrimental to the structural stability of the system, and to properly take them into account a nonlinear analysis would have been needed. The present findings (see Figures 11 and 12) confirm this importance: the bending moment transfer has a primary role in snap-buckling occurrence.

\section{Composite Materials (anisotropic case)}

Previous discussions showed that for the isotropic and orthotropic cases the driving mechanism which leads to the snap-buckling is closely tied with bending effects. It was also demonstrated that the bending stiffness ratio $D_{22}^{r}$ was an effective parameter to predict if the nonlinear response presents a snap-buckling instability. In particular, it was shown that the upper wing has to be more bending compliant to avoid the snap-buckling. It was found that when $D_{22}^{r} \equiv \frac{D_{22}^{\mathrm{LW}}}{D_{22}^{\mathrm{UW}}}$ is bigger than a critical value, then the instability disappears; $\left[D_{22}^{r}\right]_{\mathrm{CR}}$ does not represent an universal value, on the contrary, its magnitude is expected to be a case-dependent parameter.

For the same unswept joined-wing layout, the next step is the adoption of composite materials to introduce anisotropic effects and investigate how they influence the nonlinear response. In particular, two main questions are here answered. Is $D_{22}^{r}$ sufficient to describe the tendency of the structure to experience a snap-buckling? What are the effects of anisotropy on the global bending stiffness and snap-buckling?

To answer the first question, two new configurations are investigated (see Table 4). In the first one, named UANIMP1, the lower wing is isotropic and the material is the one adopted for $U R E F$ configuration. The upper wing is simulated with a multilayered orthotropic plate. The second configuration, named UANIMP2, presents a symmetric laminate for the upper wing, whereas the lower wing is made of the same isotropic reference material. Both configurations present the same value for $D_{22}^{r}$; however, the nonlinear responses are dramatically different (see Figure 14) and the configuration UANIMP2 does not experience snap-buckling. 
This qualitative investigation shows that the new coupling between the torsional deformation and bending moment plays an important role as far as the stability properties are concerned.

Figure 15 shows how the anisotropy introduces torsional deformations (configuration UANIMP2) which are not present for configuration UANIMP1.

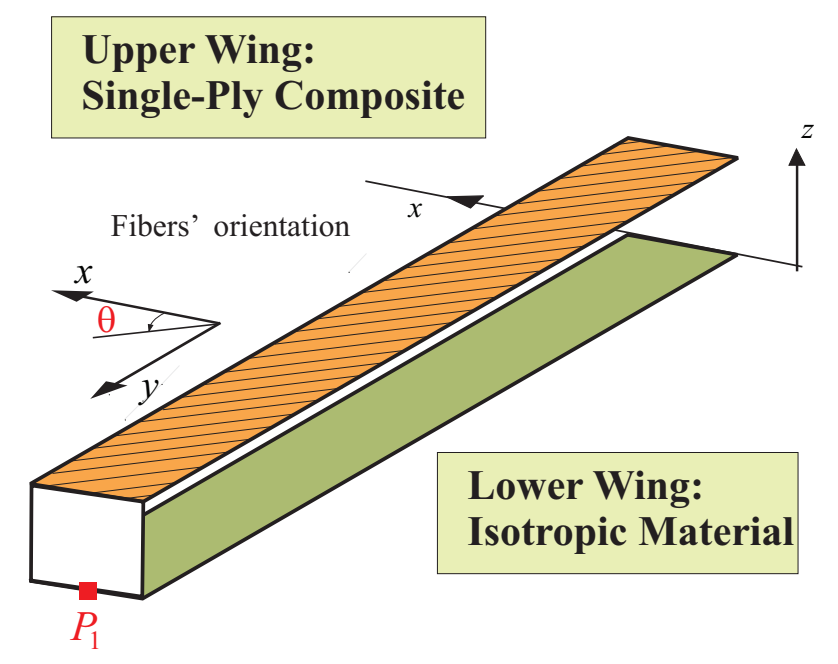

Figure 13. Unswept configurations for anisotropic cases.

A series of additional configurations have been created (see Table 5). The lower wing is isotropic and the adopted material is the one used for the UREF case. The upper wing is simulated with a single lamina whose orientation is varied according to Table 5. Results indicate (see Figure 16 and Table 5) that the bending-torsional coupling has a major role in determining when the snap-buckling occurs. This is clearly understood if for example configurations UANISP2 and UANISP 4 are compared. The two configurations do not present snap-buckling, although this was expected for the first one having $D_{22}^{r}>\left[D_{22}^{r}\right]_{\mathrm{CR}}$, it was not expected for the second one, for which $D_{22}^{r}<\left[D_{22}^{r}\right]_{\mathrm{CR}}$. Analogous situation come when comparing UANISP11 and UANISP12. Unexpected behaving cases present a value of $D_{26}^{\mathrm{UW}}$ different than zero. Since the wing system is unswept, the coupling between the torsion and bending are due only to the anisotropy of the material.

This is why for the anisotropic case understanding the mechanism which leads to the instability is more challenging.

To answer the second question (i.e., identify what are the effects of the anisotropy on global bending stiffness and snap-buckling), three different configurations (UANISP15, UANISP4, and UANISP12 [see Table 5]) are selected. None of them experiences the nonlinear buckling, and, according to Figure 17, they present high overall stiffness. The configurations UANISP 4 and UANISP12 are stiffer than the configuration UANISP15 (especially for larger values of the load step $\Lambda$ ) confirming that composite materials can be effectively used to change the structural behavior of the system. 


\begin{tabular}{|l|c|c|c|c|}
\hline \multicolumn{5}{|c|}{ Cases and relative parameters } \\
\hline Case ID & Wing & Lamination & $\begin{array}{c}\text { Ratio } \\
D_{22}^{r}=\frac{D_{22}^{\mathrm{LW}}}{D_{22}^{\mathrm{UW}}}\end{array}$ & SNAP \\
\hline UANIMP1 & Upper & $90_{0.049} / 0_{0.25} / 90_{0.402} / 0_{0.25} / 90_{0.049}$ & 2.4 & YES \\
\hline UANIMP2 & Upper & $17_{0.1} / 45_{0.8} / 17_{0.1}$ & 2.4 & NO \\
\hline
\end{tabular}

Table 4. Details about the materials used for the different configurations. For each case the lower wing is composed of the reference isotropic material, where the upper wing is composed of a composite material with plies laminated as indicate above. Each ply is manufactured with the same material $E_{1}=8.5 \cdot 10^{7}\left[\frac{\mathrm{Kg}}{\mathrm{mm} \cdot \mathrm{s}^{2}}\right], E_{2}=0.66 \cdot 10^{7}\left[\frac{\mathrm{Kg}}{\mathrm{mm} \cdot \mathrm{s}^{2}}\right], G_{12}=2.6 \cdot 10^{7}\left[\frac{\mathrm{Kg}}{\mathrm{mm} \cdot \mathrm{s}^{2}}\right], \nu_{12}=0.33$.

\begin{tabular}{|c|c|c|c|c|c|c|}
\hline \multicolumn{7}{|c|}{ Cases and relative parameters } \\
\hline Case ID & Wing & Orientation & $\begin{array}{c}\text { Ratio } \\
D_{22}^{r}=\frac{D_{22}^{\mathrm{LW}}}{D_{22}^{\mathrm{UW}}}\end{array}$ & $\begin{array}{c}D_{26}^{U W} \times 10^{-7} \\
{\left[\frac{K g \cdot m m^{2}}{s^{2}}\right]}\end{array}$ & SNAP & $\begin{array}{c}\text { UW } \\
\text { DATA }\end{array}$ \\
\hline \multirow{10}{*}{$\begin{array}{l}\text { UANISP1 } \\
\text { UANISP2 } \\
\text { UANISP3 } \\
\text { UANISP4 } \\
\text { UANISP5 } \\
\text { UANISP6 } \\
\text { UANISP7 } \\
\text { UANISP8 } \\
\text { UANISP9 } \\
\text { UANISP10 }\end{array}$} & Upper & $0^{\circ}$ & 4.5 & \multicolumn{2}{|r|}{$\mathrm{NO}$} & \multirow{10}{*}{$\begin{array}{c}E_{1}=12.5 \\
E_{2}=1.7 \\
G_{12}=2.7 \\
\nu_{12}=0.33\end{array}$} \\
\hline & Upper & $15^{\circ}$ & 3.4 & 0.09 & $\mathrm{NO}$ & \\
\hline & Upper & $30^{\circ}$ & 1.9 & 0.18 & $\mathrm{NO}$ & \\
\hline & Upper & $32.5^{\circ}$ & 1.8 & 0.19 & $\mathrm{NO}$ & \\
\hline & Upper & $35^{\circ}$ & 1.6 & 0.20 & YES & \\
\hline & Upper & $37.5^{\circ}$ & 1.5 & 0.21 & YES & \\
\hline & Upper & $45^{\circ}$ & 1.2 & 0.23 & YES & \\
\hline & Upper & $60^{\circ}$ & 0.8 & 0.22 & YES & \\
\hline & Upper & $75^{\circ}$ & 0.7 & 0.14 & YES & \\
\hline & Upper & $90^{\circ}$ & 0.6 & 0 & YES & \\
\hline UANISP11 & Upper & $45^{\circ}$ & 2.6 & 0.16 & $\mathrm{NO}$ & $E_{1}=8.5$ \\
\hline UANISP12 & Upper & $60^{\circ}$ & 1.4 & 0.20 & $\mathrm{NO}$ & $E_{2}=0.66$ \\
\hline UANISP13 & Upper & $62.5^{\circ}$ & 1.3 & 0.20 & YES & $G_{12}=0.56$ \\
\hline UANISP14 & Upper & $65^{\circ}$ & 1.3 & 0.19 & YES & $\nu_{12}=0.28$ \\
\hline UANISP15 & Upper & Isotropic & 2.6 & 0 & $\mathrm{NO}$ & $\begin{array}{l}E=2.65 \\
\nu=0.33\end{array}$ \\
\hline
\end{tabular}

Table 5. Details about the materials used for the different configurations. For each case the lower wing is made of the reference isotropic material, where the upper wing is composed of a single ply with the fibers oriented as indicate above (cases UANISP1 through UANISP14), or of an isotropic material ( $U A N I S P 15)$. The Young and shear moduli of this Table are expressed in $\left[\frac{\mathrm{Kg}}{\mathrm{mm} \cdot \mathrm{s}^{2}}\right]$ and the values reported in the Table need to be multiplied by $10^{7}$. 


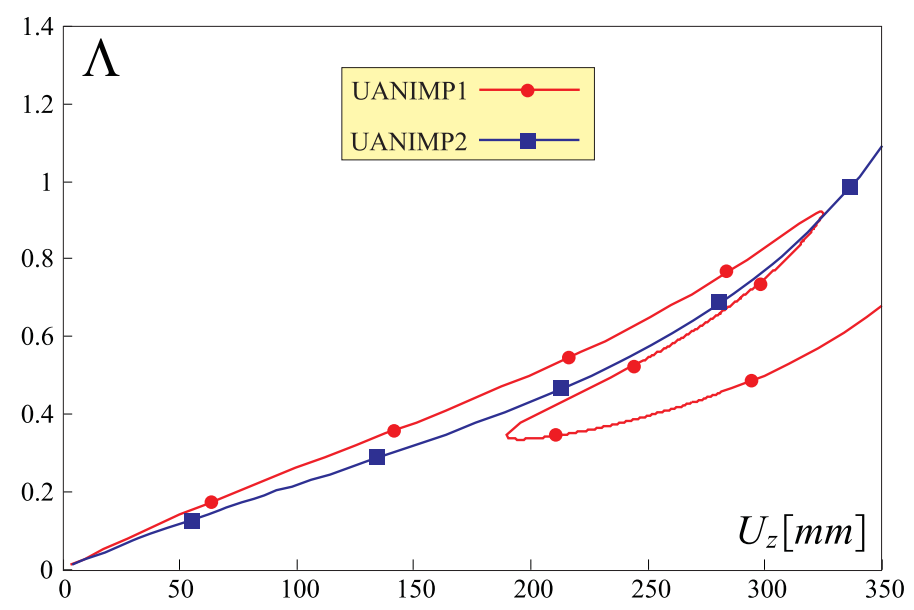

Figure 14. Cumulative vertical displacement of $P_{1}$ versus $\Lambda$ for two different configurations featuring a lower wing composed of an isotropic material and an upper wing with an orthotropic and anisotropic behaving materials, obtained through a different disposition of plies. As a consequence of the ad-hoc lamination schemes of the plies, the ratio $D_{22}^{r}$ is equal for the two cases. See Table 4 for details.

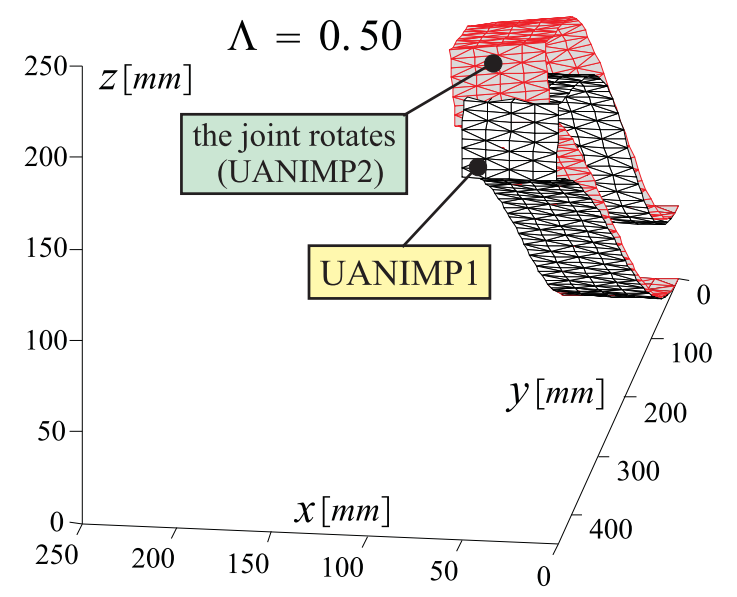

Figure 15. Comparison of the deformed configurations for configurations UANIMP1 and $U A N I M P 2$. Notice that when the anisotropy is introduced (configuration UANIMP2) there is a lateral tilting of the joint.

In the practice, the design is more challenging since it must be taken into account the structural weight and stress levels. Moreover, the actual aerodynamic loads are of a nonconservative type and the torsional-bending coupling is then even more important: the aerodynamic forces are heavily affected by a change of angle of attack (torsion) of the wing. This study is the first step in the understanding of the difficulties and challenges associated with the nonlinear response for the case of anisotropic Joined Wings. 

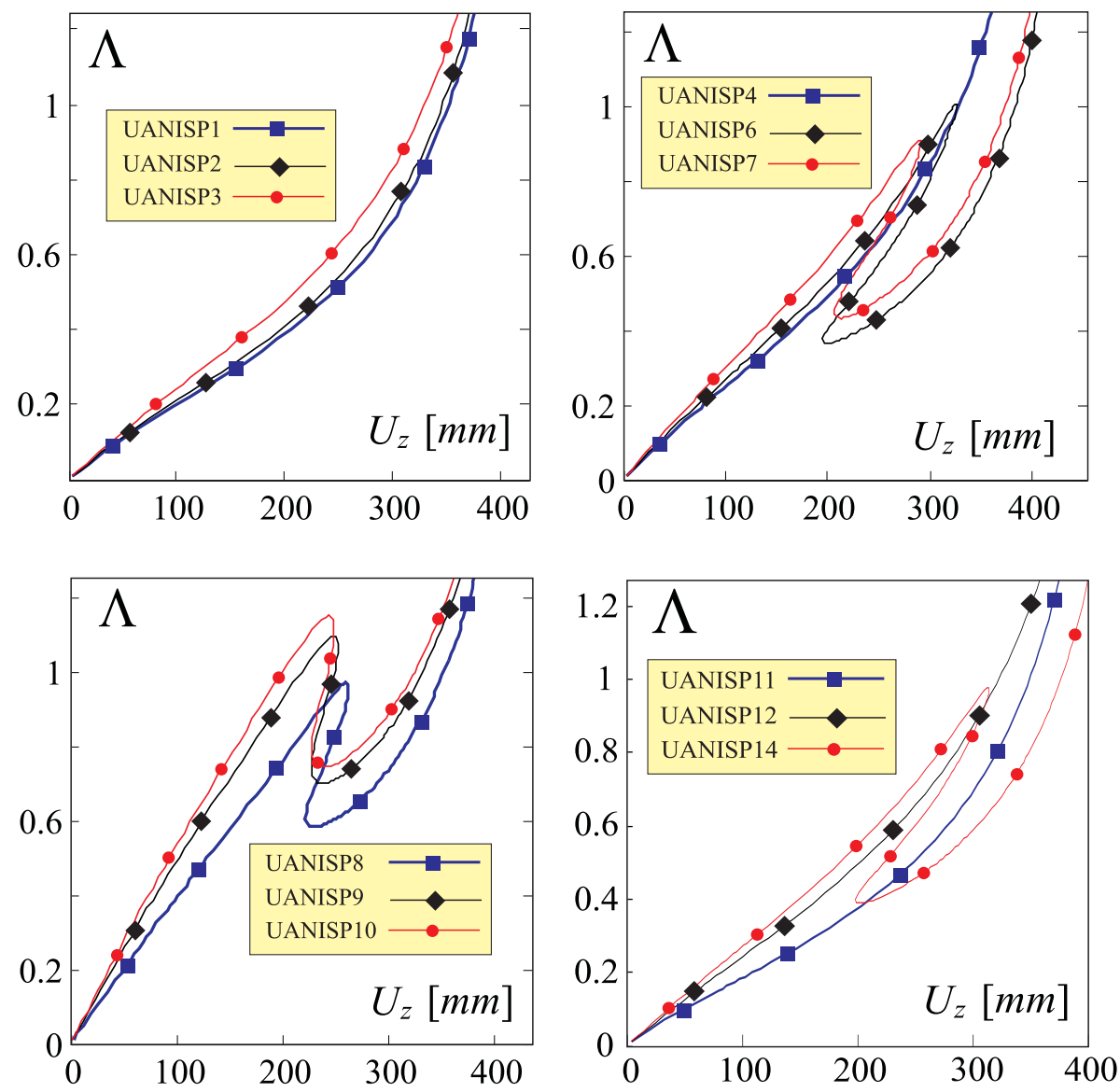

Figure 16. Responses for different configurations. The lower wing is composed of the reference isotropic material $\left(E=E_{\mathbf{R E F}}\right.$ and $\left.\nu=\nu_{\mathbf{R E F}}\right)$ for all the cases, the upper wing is composed of a single layer with $E_{1}^{\mathrm{UW}}=12.5 \cdot 10^{7}\left[\frac{\mathrm{Kg}}{\mathrm{mm} \cdot \mathrm{s}^{2}}\right], E_{2}^{\mathrm{UW}}=1.7 \cdot 10^{7}\left[\frac{\mathrm{Kg}}{\mathrm{mm} \cdot \mathrm{s}^{2}}\right], G_{12}^{\mathrm{UW}}=2.7 \cdot 10^{7}\left[\frac{\mathrm{Kg}}{\mathrm{mm} \cdot \mathrm{s}^{2}}\right]$ and $\nu_{12}^{\mathrm{UW}}=0.33$. The orientation of the fibers for each case is specified in Table 5.

\section{Swept Joined Wings and Composites}

From the analysis of unswept Joined Wings two main concepts could be identified. First, the ratio between the bending stiffness of the wings is an important parameter to establish if the snap-buckling occurs. In particular, the upper wing has to be more bending compliant than the lower wing to remove the instability.

Second, the anisotropy introduces a coupling between the torsion and bending which is not present in isotropic unswept Joined Wing. This coupling modifies the snap-buckling occurrence.

A similar study is now attempted for the swept Joined Wings (see Figure 2). It is necessary to investigate this case since even when isotropic materials are used, a coupling between the bending and torsion due to the geometry of the wing system arises. It may also be observed (see Figure 2) that the sweep angle is moderately low. It is then reasonable to expect that the snap occurrence is still regulated by bending stiffness related parameters. 


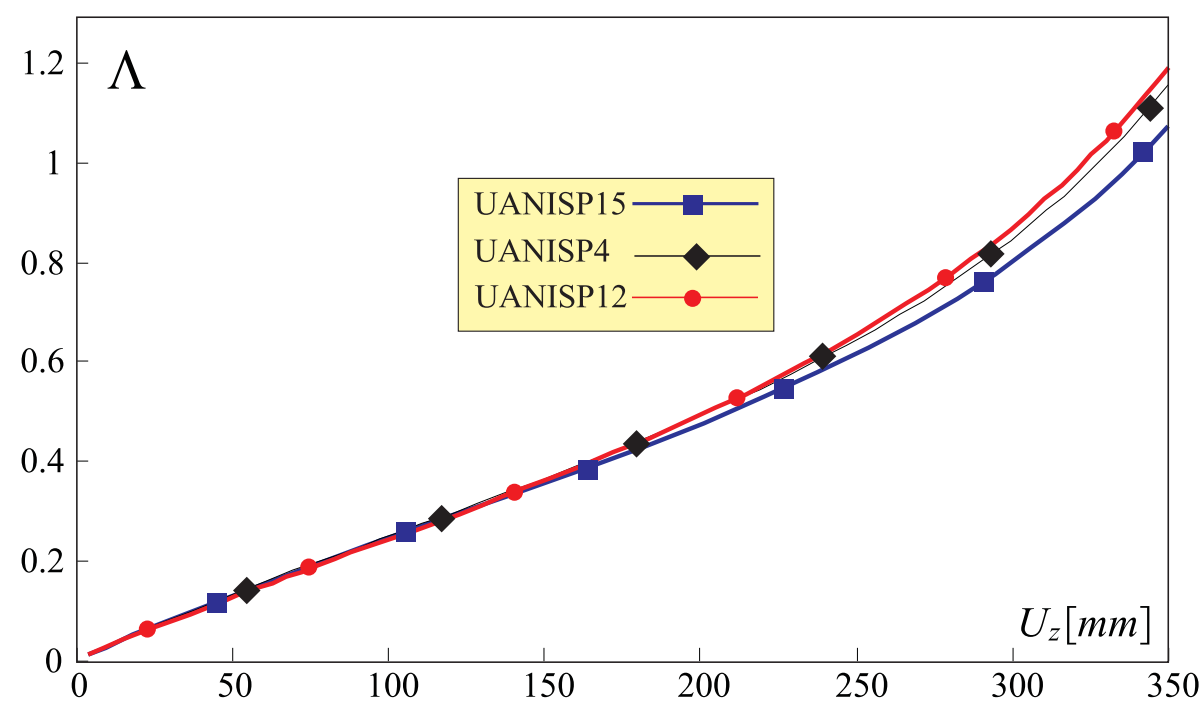

Figure 17. Comparison of responses for configurations UANISP4, UANISP12 and UANISP15. All the three configurations have a lower wing composed of the isotropic reference material. Configuration UANISP15 features an upper wing of a different isotropic material, where UANISP4 and UANISP12 have an upper wing composed of a single ply composite of two different materials. Details about the materials are reported in Table 5. Given the materials, the three configurations represent the stiffest responses which do not show buckling.

In order to better investigate the physics related to the bending, it is useful to introduce two local coordinate systems, one for each wing. The direction of the $z$-axis remains parallel to the global $z$-axis, whereas the local $y$-axis runs along the wing-span direction. In such a way the terms of the $\boldsymbol{D}$ matrices for the upper and lower wings maintain an immediate physical interpretation. Figure 2 clarifies the orientation of the lower and upper wing local axes.

\section{A. Effects of Lower-to-Upper-Wing Stiffness Ratio}

\section{Isotropic case}

The ratio of the Young's moduli of the two wings is varied. However, Young's moduli are selected so that the initial slopes of the displacements are the same (the initial slope is related to the stiffness of the linear analysis). The details about the materials of each configuration are shown in Table 6 and Figure 18, and the graphs of the cumulative vertical displacement of point $P_{1}$ are presented in Figure 19. It can be inferred that, as in the unswept case, the ratio $E^{r}=\frac{E^{\mathrm{LW}}}{E^{\mathrm{UW}}}$ has an important role. However, the required value for avoiding snap is considerably larger (see Table 6) than the one needed for the unswept wings case (see Table 1). This means that the lower wing has to be much stiffer than the upper wing in order to avoid snap-buckling.

It is possible to observe that each wing of the swept configuration results to be a slightly 


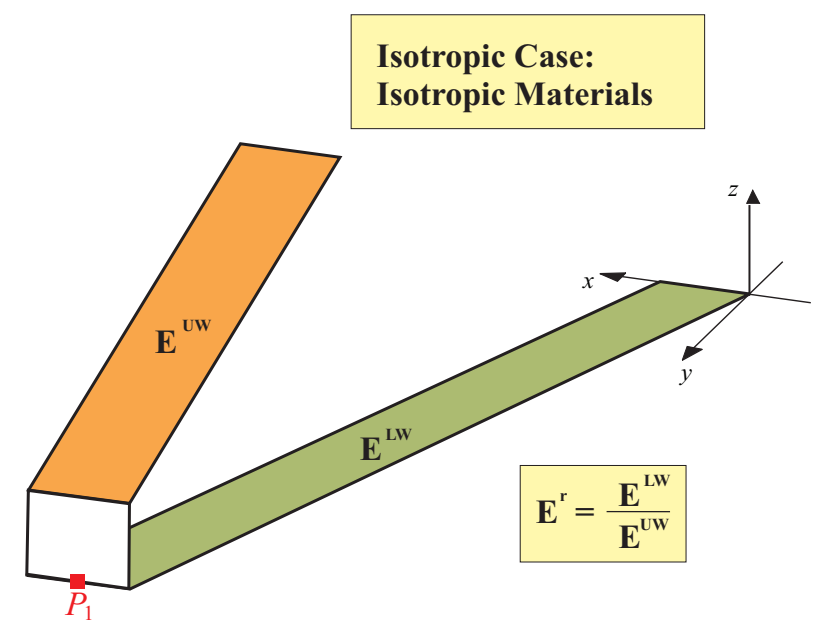

Figure 18. Swept configurations for isotropic cases and definition of lower-to-upper wing stiffness ratio.

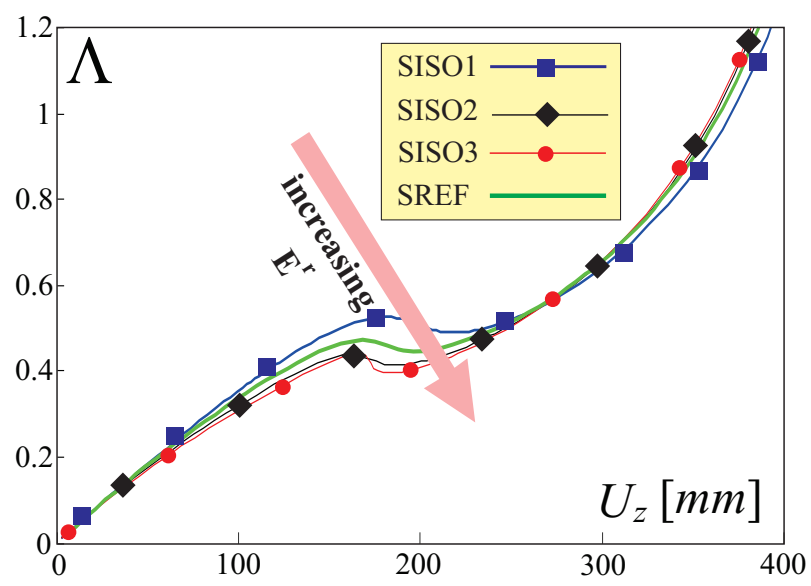

(a)

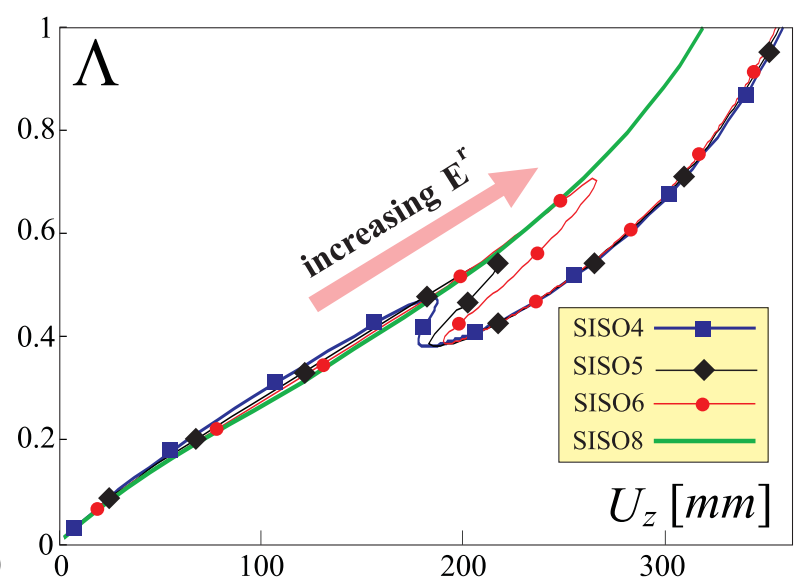

(b)

Figure 19. Load parameter $\Lambda$ versus cumulative vertical displacement $U_{z}$ of lower wing's tip point $P_{1}$ for configurations employing different isotropic materials (see Table 6). The ratio of Young's Modulus of the upper wing to the one of the lower wing is varied but the initial rate of change (linear case) of cumulative vertical displacement of $P_{1}$ is constant.

longer and leaner (higher aspect ratio) than the previous unswept cases. However, since the sweep angle is small, the aspect ratio is not significantly affected. The consistent difference of the critical ratio $E^{r}$ found for the swept case could be thought to come mainly from effects introduced by the torsion.

Analyses of two configurations ${ }^{32}$, one incurring in snap, SISO5, and one not, SISO8, for two different load conditions are depicted in Figures 20 and 21. For the load level $\Lambda=0.5$ SISO5 is not very far to buckle, however, the two deformed shapes are almost superimposed (see Figure 20), except for the upper wings. In the SISO5 case the upper wing experiences a more pronounced inward bending deformation, similarly with what was found for the swept cases. 


\begin{tabular}{|c|c|c|c|c|}
\hline \multicolumn{5}{|c|}{ Cases and relative parameters } \\
\hline Case ID & Wing & $\begin{array}{l}\text { Young's Modulus } \\
E \times 10^{-7}\left[\frac{\mathrm{Kg}}{\mathrm{mm} \cdot \mathrm{s}^{2}}\right]\end{array}$ & $\begin{array}{c}\text { Ratio } \\
E^{r}=\frac{E^{L W}}{E^{U W}}\end{array}$ & Snap \\
\hline \multirow{2}{*}{ SREF } & Upper & 6.9 & \multirow{2}{*}{ 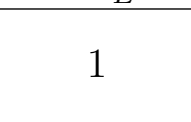 } & \multirow{2}{*}{ YES } \\
\hline & Lower & 6.9 & & \\
\hline \multirow{2}{*}{ SISO1 } & Upper & 12 & \multirow{2}{*}{0.2} & \multirow{2}{*}{ YES } \\
\hline & Lower & 2.5 & & \\
\hline \multirow{2}{*}{ SISO2 } & Upper & 5 & \multirow{2}{*}{1.8} & \multirow{2}{*}{ YES } \\
\hline & Lower & 8.9 & & \\
\hline \multirow{2}{*}{ SISO3 } & Upper & 4 & \multirow{2}{*}{2.5} & \multirow{2}{*}{ YES } \\
\hline & Lower & 10.1 & & \\
\hline \multirow{2}{*}{ SISO4 } & Upper & 3 & \multirow{2}{*}{3.8} & \multirow{2}{*}{ YES } \\
\hline & Lower & 11.3 & & \\
\hline \multirow{2}{*}{ SISO5 } & Upper & 2.5 & \multirow{2}{*}{4.8} & \multirow{2}{*}{ YES } \\
\hline & Lower & 12.0 & & \\
\hline \multirow{2}{*}{ SISO6 } & Upper & 2.2 & \multirow{2}{*}{5.6} & \multirow{2}{*}{ YES } \\
\hline & Lower & 12.4 & & \\
\hline \multirow{2}{*}{ SISO7 } & Upper & 2.1 & \multirow{2}{*}{6.0} & \multirow{2}{*}{ YES } \\
\hline & Lower & 12.5 & & \\
\hline \multirow{2}{*}{ SISO8 } & Upper & 2.0 & \multirow{2}{*}{6.3} & \multirow{2}{*}{$\mathrm{NO}$} \\
\hline & Lower & 12.6 & & \\
\hline
\end{tabular}

Table 6. Details about the isotropic materials used for the different swept Joined Wings. The Poisson ratio is $\nu=0.33$ for each case.

The load level $\Lambda=0.6$ represents a post-buckling situation for the configuration SISO5, as it could be verified in Figure 19(b). Besides experiencing an almost rigid rotation along $x$-axis, in this case the joint undergoes a negative rotation along global $y$-axis as well (see Figure 21).

In conclusion, the interactions between the wings are more complicated in the case of swept Joined Wings even when isotropic materials are used. This is due to the rise of forces inherent to the geometrical layout which couples the bending and torsional effects. These forces have an important role in influencing the snap phenomenon: although the span-wise bending actions drive the instability phenomenon, torsion contributes to regulate it. For example, compared to the unswept isotropic cases, the lower wing has to be significantly stiffer in order snap to be avoided. This could be intuitively explained as follows. Figure 21 shows that when the instability takes place a significant rigid rotation of the joint is experienced (SISO5 configuration). Thus, the high stiffness is "required" to counteract also this joint's rotation and thus, to avoid the snap-bucking. 


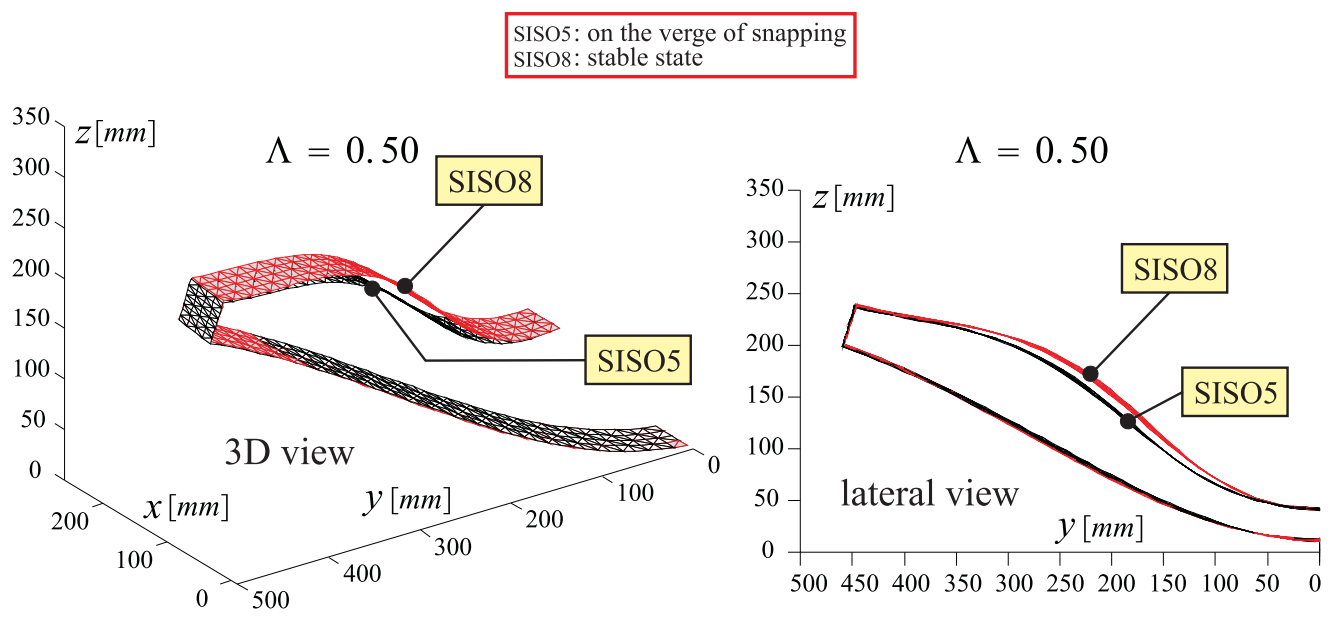

Figure 20. Comparison of deformed configurations SISO5 and SISO8 at $\Lambda=0.5$. Tridimensional and side view.

SISO5: post-critical state SISO8: stable state

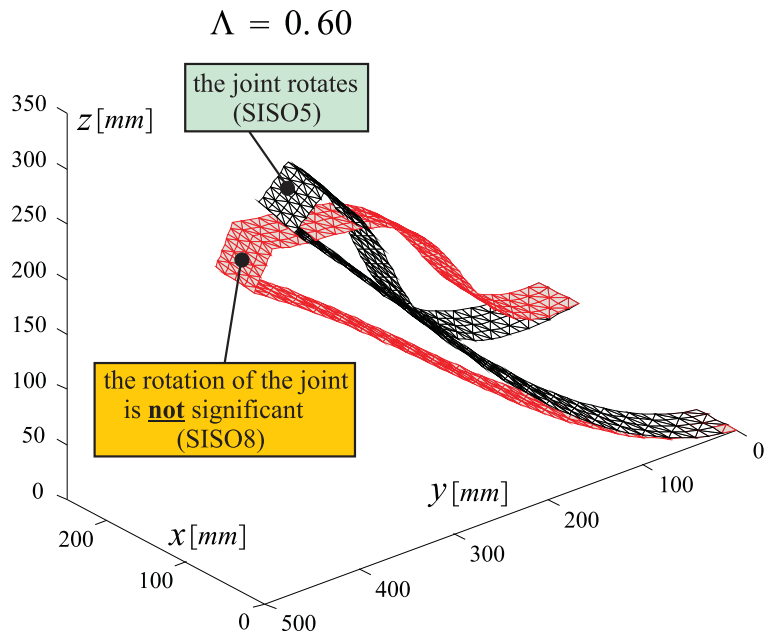

$\Lambda=0.60$

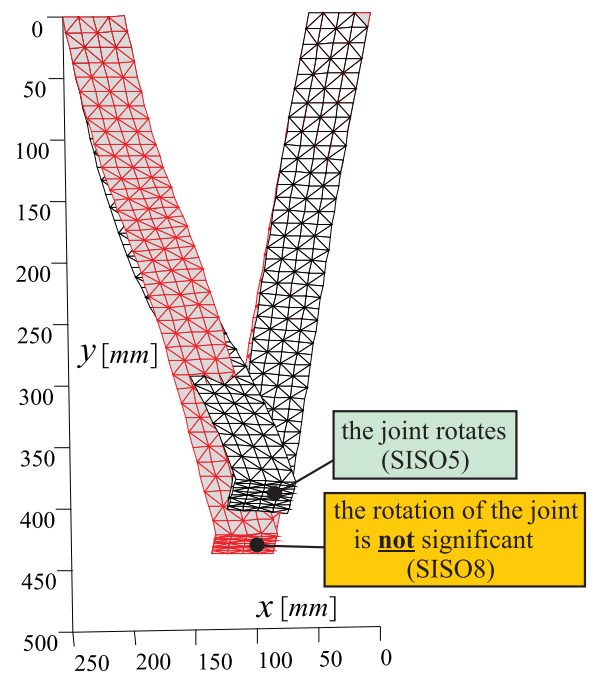

Figure 21. Comparison of configurations SISO5 and SISO8 at $\Lambda=0.6$. Tridimensional and upper view. The SISO5 is in its post-snap configuration.

\section{Orthotropic Cases}

The introduction of an orthotropic material for the wings allows not only to differentiate between the relative importance of the Young's moduli $E_{1}$ and $E_{2}$, but gives also the valuable possibility to isolate and study more in depth the torsional effects. This was not possible for unswept layouts since, featuring the configuration geometrical symmetry, no torsional effects were introduced in the structure.

The stiffness parameter $D_{66}$ is now varied to explore the effects due to the torsion but the bending stiffness ratio, extensively discussed in the preceding sections, is not modified. The 


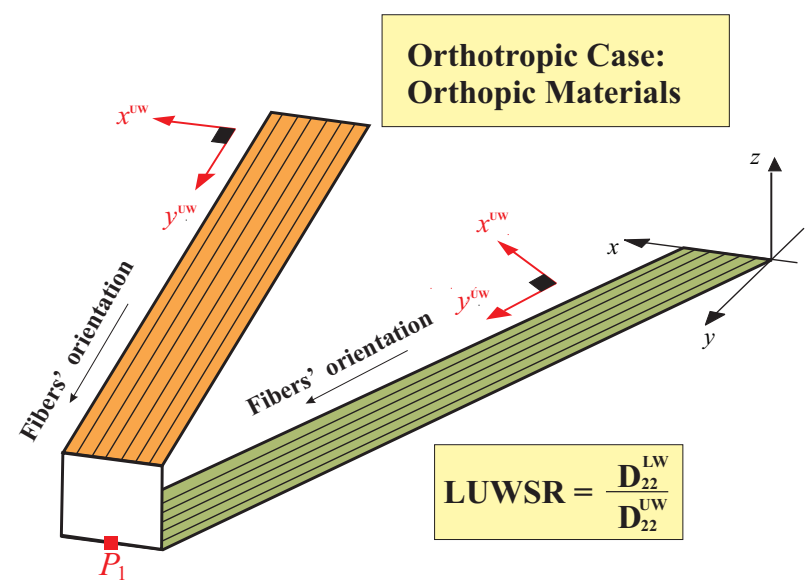

Figure 22. Swept configurations for orthotropic cases and definition of lower-to-upper wing stiffness ratio.

\begin{tabular}{|c|c|c|c|c|}
\hline \multicolumn{5}{|c|}{ Cases and relative parameters } \\
\hline \multirow{2}{*}{ Case ID } & \multirow{2}{*}{ Wing } & Shear Modulus & \multirow{2}{*}{$\begin{array}{c}\text { Ratio } \\
E_{1}^{r}=\frac{E_{1}^{L W}}{E_{1}^{U W}}\end{array}$} & \multirow{2}{*}{ Snap } \\
\hline & & $G_{12} \times 10^{-7}\left[\frac{\mathrm{hg}}{\mathrm{mm} \cdot \mathrm{s}^{2}}\right]$ & & \\
\hline \multirow{2}{*}{ SREF } & Upper & 2.6 & \multirow{2}{*}{1} & \multirow{2}{*}{ YES } \\
\hline & Lower & 2.6 & & \\
\hline \multirow{2}{*}{ SORTHO1 } & Upper & 120 & \multirow{2}{*}{1} & \multirow{2}{*}{ YES } \\
\hline & Lower & 120 & & \\
\hline \multirow{2}{*}{ SORTHO2 } & Upper & 300 & \multirow{2}{*}{1} & \multirow{2}{*}{ YES } \\
\hline & Lower & 300 & & \\
\hline \multirow{2}{*}{ SORTHO3 } & Upper & 600 & \multirow{2}{*}{1} & \multirow{2}{*}{ YES } \\
\hline & Lower & 600 & & \\
\hline \multirow{2}{*}{ SORTHO4 } & Upper & 1500 & \multirow{2}{*}{1} & \multirow{2}{*}{ YES } \\
\hline & Lower & 1500 & & \\
\hline \multirow{2}{*}{ SORTHO5 } & Upper & 2500 & \multirow{2}{*}{1} & \multirow{2}{*}{$\mathrm{NO}$} \\
\hline & Lower & 2500 & & \\
\hline
\end{tabular}

Table 7. Details about the orthotropic materials used for the different swept Joined Wings. The Young's Moduli and Poisson ratio are $E_{1}=E_{2}=E_{\mathbf{R E F}}$ and $\nu_{12}=\nu_{\mathbf{R E F}}$ for each wing of all cases. Thus, every considered configuration has the ratio $D_{22}^{r}$ equal to 1 .

use of orthotropic material gives the possibility to act on the $D_{66}$ coefficient maintaining the same bending stiffness ratio. In fact, as it could be inferred from its well known definition, the $D_{66}$ parameter could be fine-tuned through the adoption of different values of the material's shear modulus $G_{12}$. Moreover, this last material property does not influence the remaining parameters of the stiffness matrix $\boldsymbol{D}$. It is thus straightforward to selectively evaluate the importance of torsional deformation on the system response.

In this regard, $S R E F$ is chosen as starting configuration. Then for each wing the Young's 
moduli and Poisson's ratio are set such that:

$$
\begin{aligned}
E_{1}^{\mathrm{UW}}=E_{2}^{\mathrm{UW}} & =E_{1}^{\mathrm{LW}}=E_{2}^{\mathrm{LW}}=E_{\mathrm{REF}} \\
\nu_{12}^{\mathrm{UW}} & =\nu_{12}^{\mathrm{LW}}=\nu_{\mathrm{REF}}
\end{aligned}
$$

Different values of $G_{12}^{\mathrm{UW}}$ and $G_{12}^{\mathrm{LW}}$ are chosen, with a direct effect on $D_{66}^{\mathrm{UW}}$ and $D_{66}^{\mathrm{LW}}$ respectively, as shown in Table 7 and Figure 22. The responses are depicted in Figure 23. It could

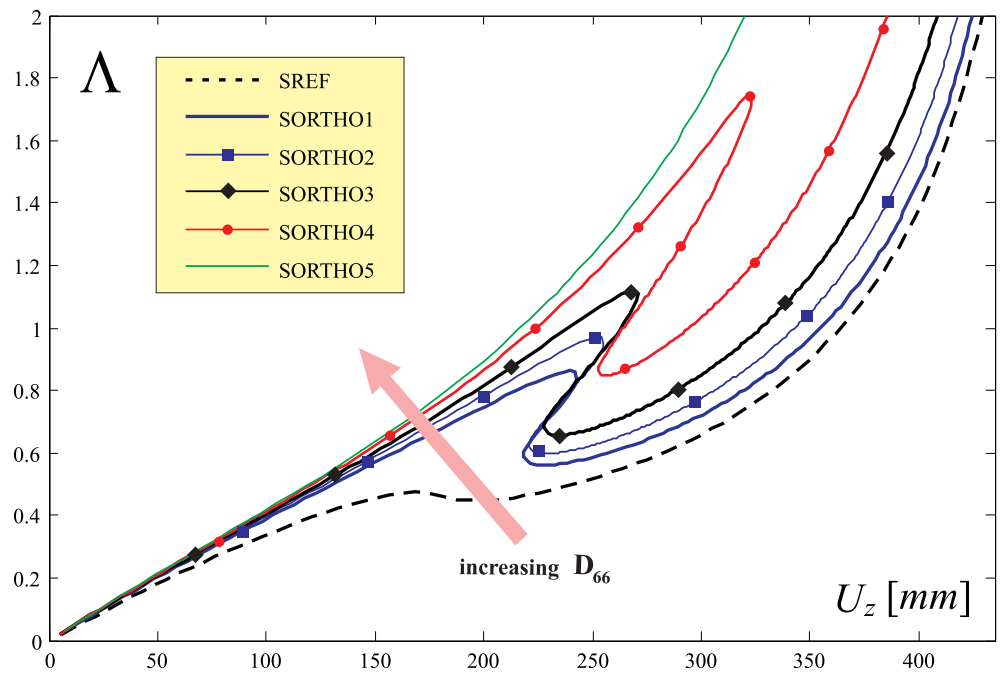

Figure 23. Cumulative vertical displacement $\left(U_{z}\right)$ of point $P_{1}$ versus load parameter $(\Lambda)$ for swept Joined Wings featuring different orthotropic wings. For each configuration upper and lower wings are manufactured with the same material. See Table 7 for details.

be inferred that torsional stiffness has an important effect on the behavior of the system. If it is increased, torsional deformations are smaller, and thus, ideally, the response is expected to be qualitatively similar to the one of the unswept case (with isotropic or orthotropic wings). This is supported noticing that $S O R T H O$ cases response is qualitatively similar to $U R E F$ 's one (see Reference [32]), showing a more abrupt snap-buckling occurrence, that is, the instability occurs without a progressive previous loss of stiffness.

As could be seen in Figure 23 if torsional stiffness is increased to a certain extent no instability phenomenon occurs, differently than the unswept case where it was not possible to eliminate buckling when $D_{22}^{r}<\left[D_{22}^{r}\right]_{\mathrm{CR}}$. This difference is a consequence of the structure's layout and overconstrained nature of the system. In practice, in cases such as SORTHO5 the torsional stiffness has a dominant role on the bending mode through the geometrical coupling, and this interaction limits the pertinence of considering the similarity between swept and unswept case.

Summarizing, in this particular overconstrained system in which bending and torsion are also coupled as a consequence of the sweep angle, it is very difficult to separate the effects on the structural response driven by one or the other deformation. 


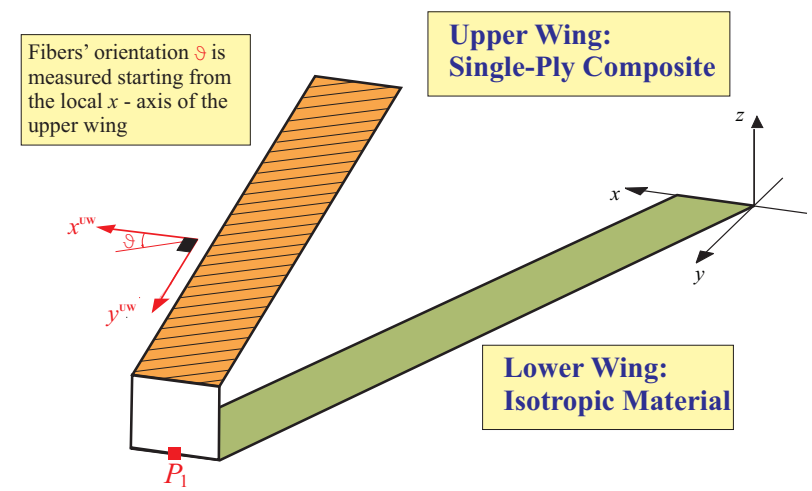

Figure 24. Swept configurations for anisotropic cases.

In this section, the lower wing is assumed to be made of the same isotropic material used for the UREF configuration. However, the upper wing is now composed of a single lamina. The fibers' orientation is measured starting from the upper wing local axis $x^{\mathrm{UW}}$, as depicted in Figure 2 and 24. Table 8 reports the configurations used to assess the anisotropic effects for the swept Joined Wing. It may be inferred that the influence of torsion is now of primary importance. In fact, some configurations featuring a relatively small value of $D_{22}^{r}$ do not present any snap-buckling phenomenon. It is relevant to investigate the effect of the sign for $D_{26}^{\mathrm{UW}}$ for practically unchanged $D_{22}^{r}$ parameters. For example, configuration SANISP16 (for which $D_{22}^{r}=1.39$ and $D_{26}^{\mathrm{UW}}=-0.20\left[\frac{\mathrm{Kg} \cdot \mathrm{mm}^{2}}{\mathrm{~s}^{2}}\right]$ ) does not experience buckling whereas configuration SANISP6 (for which $D_{22}^{r}=1.30$ and $D_{26}^{\mathrm{UW}}=+0.20\left[\frac{\mathrm{Kg} \cdot \mathrm{mm}^{2}}{\mathrm{~s}^{2}}\right]$ ) does experience instability.
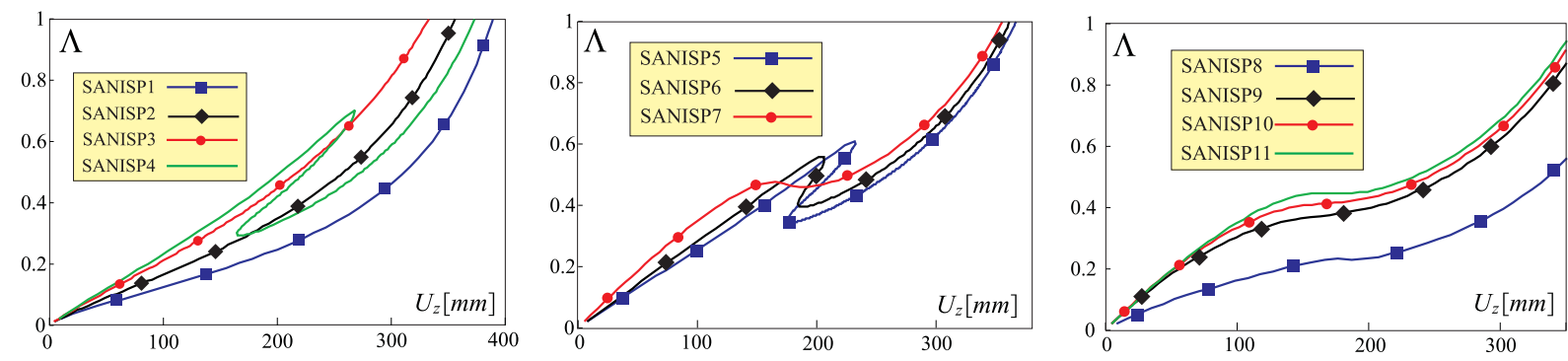

Figure 25. Cumulative vertical displacement $\left(U_{z}\right)$ of point $P_{1}$ versus load parameter $(\Lambda)$ for swept Joined Wings featuring different single-layer upper wing. See Table 8 for details.

Not all of the configurations reported in Table 8 experience buckling. It is then of a practical importance to identify the conditions for which buckling occurs from a graphical point of view. In particular, the so called Snap Buckling Region SBR for Joined Wings ${ }^{27}$ can be seen in Figure 26. It should be noted that SBR is actually the union of two subregions which do not present symmetry with respect to the zero angle. This is expected since the 
Accepted manuscript of the paper published on AIAA Journal

DOI: https://doi.org/10.2514/1.J052242

\begin{tabular}{|l|c|c|c|c|c|}
\hline \multicolumn{7}{|c|}{ Cases and relative parameters } \\
\hline Case ID & Wing & $\begin{array}{c}\text { Orientation: Angle } \\
\text { measured from } x^{\mathrm{UW}}(x)\end{array}$ & $\begin{array}{c}\text { Ratio } \\
D_{22}^{r}=\frac{D_{22}^{\mathrm{LW}}}{D_{22}^{\mathrm{UW}}}\end{array}$ & $\begin{array}{c}D_{26}^{U W} \times 10^{-7} \\
{\left[\frac{K g \cdot m m^{2}}{s^{2}}\right]}\end{array}$ & SNAP \\
\hline SANISP1 & Upper & $-11.3^{\circ}\left(0^{\circ}\right)$ & 10.71 & -0.01 & NO \\
\hline SANISP2 & Upper & $33.7^{\circ}\left(45^{\circ}\right)$ & 4.60 & 0.10 & NO \\
\hline SANISP3 & Upper & $48.7^{\circ}\left(60^{\circ}\right)$ & 2.22 & 0.18 & NO \\
\hline SANISP4 & Upper & $53.7^{\circ}\left(65^{\circ}\right)$ & 1.80 & 0.20 & YES \\
\hline SANISP5 & Upper & $58.7^{\circ}\left(70^{\circ}\right)$ & 1.51 & 0.20 & YES \\
\hline SANISP6 & Upper & $63.7^{\circ}\left(75^{\circ}\right)$ & 1.30 & 0.20 & YES \\
\hline SANISP7 & Upper & $78.7^{\circ}\left(90^{\circ}\right)$ & 0.97 & 0.11 & YES \\
\hline SANISP8 & Upper & $-56.3^{\circ}\left(-45^{\circ}\right)$ & 1.64 & -0.20 & YES \\
\hline SANISP9 & Upper & $-86.3^{\circ}\left(-75^{\circ}\right)$ & 0.91 & -0.04 & NO \\
\hline SANISP10 & Upper & $88.7^{\circ}\left(-80^{\circ}\right)$ & 0.91 & 0.01 & NO \\
\hline SANISP11 & Upper & $83.7^{\circ}\left(-85^{\circ}\right)$ & 0.92 & 0.07 & YES \\
\hline SANISP12 & Upper & $-41.3^{\circ}\left(-30^{\circ}\right)$ & 3.13 & -0.15 & NO \\
\hline SANISP13 & Upper & $-71.3^{\circ}\left(-60^{\circ}\right)$ & 1.09 & -0.17 & NO \\
\hline SANISP14 & Upper & $73.7^{\circ}\left(85^{\circ}\right)$ & 1.04 & 0.15 & YES \\
\hline SANISP15 & Upper & $-66.3^{\circ}\left(-55^{\circ}\right)$ & 1.21 & -0.19 & NO \\
\hline SANISP16 & Upper & $-61.3^{\circ}\left(-50^{\circ}\right)$ & 1.39 & -0.20 & NO \\
\hline SANISP17 & Upper & $-51.3^{\circ}\left(-40^{\circ}\right)$ & 1.99 & -0.19 & YES \\
\hline SANISP18 & Upper & $-46.3^{\circ}\left(-35^{\circ}\right)$ & 2.47 & -0.17 & YES \\
\hline
\end{tabular}

Table 8. Test cases. For each case the lower wing is made of the reference isotropic material, where the upper wing is a single ply. The material used for the upper wing presents $E_{1}^{\mathrm{UW}}=$ $8.5 \cdot 10^{7}\left[\frac{\mathrm{Kg}}{\mathrm{mm} \cdot \mathrm{s}^{2}}\right], E_{2}^{\mathrm{UW}}=0.66 \cdot 10^{7}\left[\frac{\mathrm{Kg}}{\mathrm{mm} \cdot \mathrm{s}^{2}}\right], G_{12}^{\mathrm{UW}}=0.56 \cdot 10^{7}\left[\frac{\mathrm{Kg}}{\mathrm{mm} \cdot \mathrm{s}^{2}}\right], \nu_{12}^{\mathrm{UW}}=0.28$. The fibers' angle is measured starting from the upper wing's local coordinate system $x^{\mathrm{UW}}$. In parenthesis the same fibers' angle is referred to the global coordinate system $x$-axis (see Figure 25).

Joined Wing and its materials do not present symmetries and the complex bending-torsion coupling affects the nonlinear response in a non-trivial manner.

Figure 25 shows the responses relative to some of the cases reported in Table 8.

Figure 27 shows graphically the connection between typical responses and fibers' orientation. Some observations could be stated. When the fibers are directed along the upper wing's chord-wise direction (local x-axis) the response is not very stiff, and snap-buckling does not occur. This is not surprising since the lower-to-upper wing bending stiffness ratio has a large value (see for example configuration SANISP1 in Table 8: $D_{22}^{r}$ is 10.71). It is also expected that if the fibers are rotated, the structure exhibits overall a stiffer response, since material is properly oriented to counteract the flexional actions. However, this is not always the case. If the fibers are rotated in the positive $\vartheta$ direction, the response is quite 


\section{$S B R=S B R_{I} \cup S B R_{I I}$}

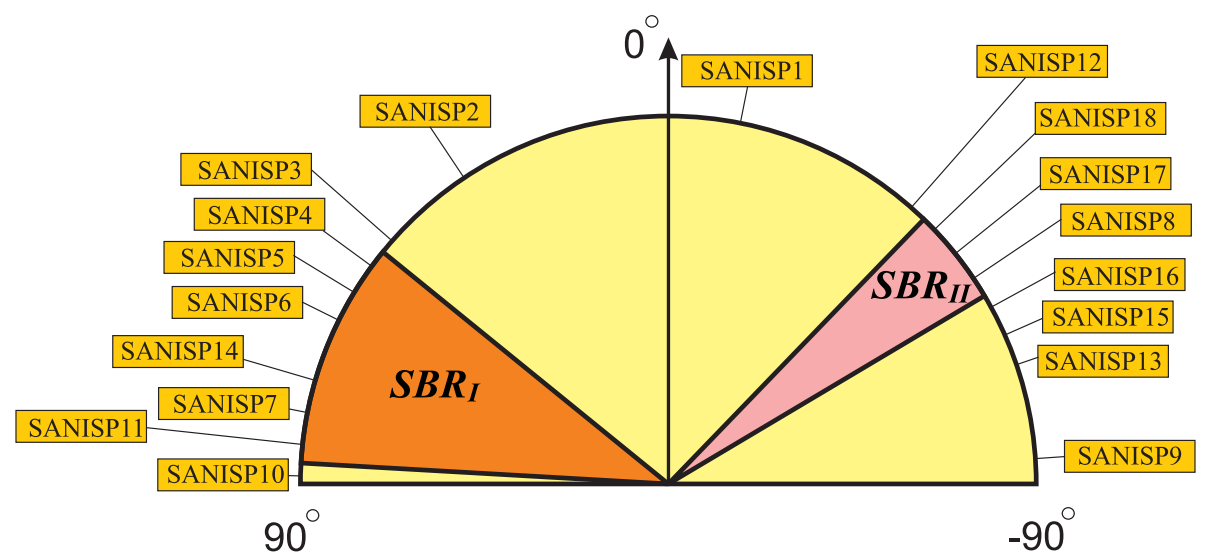

Figure 26. Snap Buckling Region for configurations with different orientation of the upper wing's fibers (the angles are measured in the upper wing's local coordinate system). The configurations in the $S B R$ experience buckling.

stiffer (see for example SANISP3 in Figure 27), whereas if they are rotated in the opposite direction the response does not only contradict the intuition based on a stiffness perspective, but with a further decrease of $\vartheta$ a snap phenomenon occurs (see SANISP12, SANISP18, and SANISP8 in Figure 27). It is not trivial to interpret and fully understand this behavior. The bending-torsion coupling at both geometrical and material level for these cases is unfavorable, and gives rise to configurations which are compliant in resisting the load and could even undergo instability.

With a further decrease of $\vartheta$, an interesting behavior is detected. Comparing SANISP18 and SANISP8, both having buckling problem, a main difference is observed: SANISP18 corresponds to an abrupt buckling, whereas SANISP8 has a mild snap, that is, in the whole neighborhood of the limit point, there is loss in stiffness. If the fibers' orientation is decreased to the point that buckling disappears, as for example happens with configuration SANISP16, this local softening is still observed.

Notice that the initial response has not significantly changed from the SANISP1 case, although the fibers' orientation has varied of $50^{\circ}$.

The pattern showed in SANISP16 is maintained if the angle is further decreased. But, the structural response gains progressively stiffness, until the fibers are oriented approximately along the span-wise direction, SANISP10. Based on the previous gained experience about stiffness ratio influence on snap occurrence, it is unexpected that this configuration does not show a snap-buckling phenomenon, although the local loss of stiffness pattern is practically as inconvenient as an instability.

If starting from $\vartheta=0^{\circ}$ the angle is increased the behavior is somehow expected. In fact, 


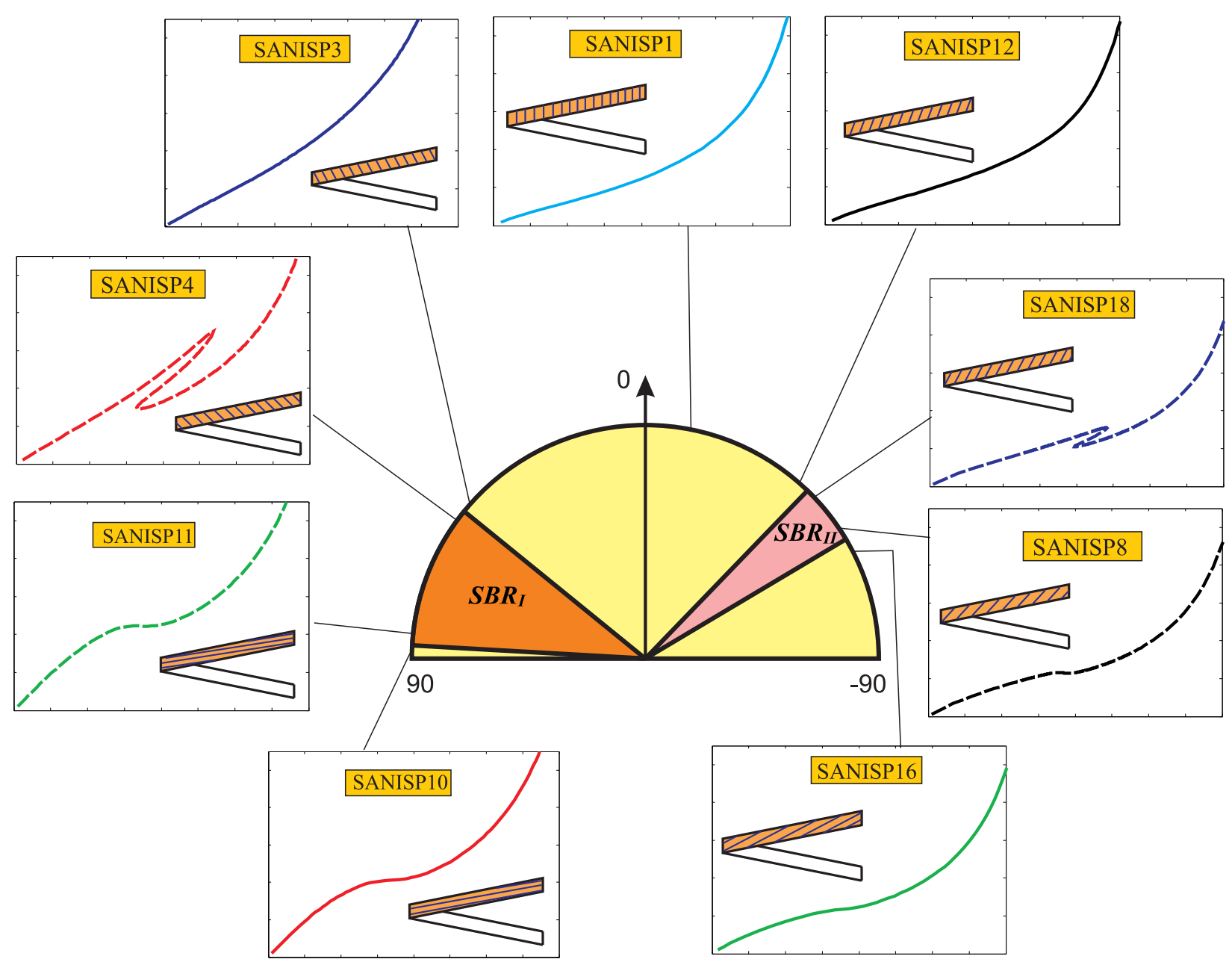

Figure 27. Snap Buckling Region and typical responses (vertical displacement of point $P_{1}$ versus load factor).

stiffness of the response increases, SANISP3 and snap-buckling eventually occurs, SANISP4. Notice that this snap has the usual behavior with the limit point occurring without having any loss of stiffness in the load-displacement curve preceding the limit point. Now, a further increase in fibers' angle leads to an initial increase in stiffness, followed however by an snap-buckling instability at a lower load level. The instability is characterized now (see SANISP11) by a local softening in the limit point region (mild snap-buckling), similar to what observed for SANISP8 configuration.

Unexpectedly a further increase in $\vartheta$ leads to a response not showing a snap-buckling phenomenon, but, the local softening is still present (SANISP10).

Summarizing, starting from $\vartheta=0$, if the angle is changed in the positive direction the response becomes stiffer and snap-buckling occurs. Then, due probably to torsional effects, local softening is introduced in the neighborhood of the limit point region. If the angle is changed in the negative direction the response experiences instability without being initially 
stiffer. In this case the bending-torsion coupling counteracts the typical stiffer behavior related with smaller $D_{22}^{r}$ (and same $D_{22}^{\mathrm{LW}}$ ), but does not avoid snap occurrence. Finally, when the fibers are oriented nearly along the span-wise direction, no instability is present, although there is a pronounced loss of stiffness.

It is the authors' opinion that further studies are needed in order to better understand the phenomenon at its basic level. In fact, although in this case the structural response is basically regulated by the bending-torsion coupling, the strong nonlinear behavior represents an added complication and makes extremely difficult and possibly unsuccessful the attempt to explain in detail the essence of the problem breaking it down in simpler cases.

\section{B. Joint's Height Effects in the Case of Anisotropic Swept Joined Wings}

In Reference [27] it was demonstrated that for the case isotropic materials the increase of the height of the joint was beneficial. This is confirmed in this work for the case of composite materials, as clearly shown in Figure 28 where SANISP4 and SANISP11 (see also Table 8) are presented. A joint's height larger than a value $\left(b=\frac{65}{50} a\right.$ and $b=\frac{50}{50} a$ for the cases of Figure 28(a) and 28(b) respectively) leads to a stiffer-snap-buckling-free response. This is the concept of Upper Limit Snap Buckling Region (ULSBR) introduced in Reference [27] for the isotropic case.

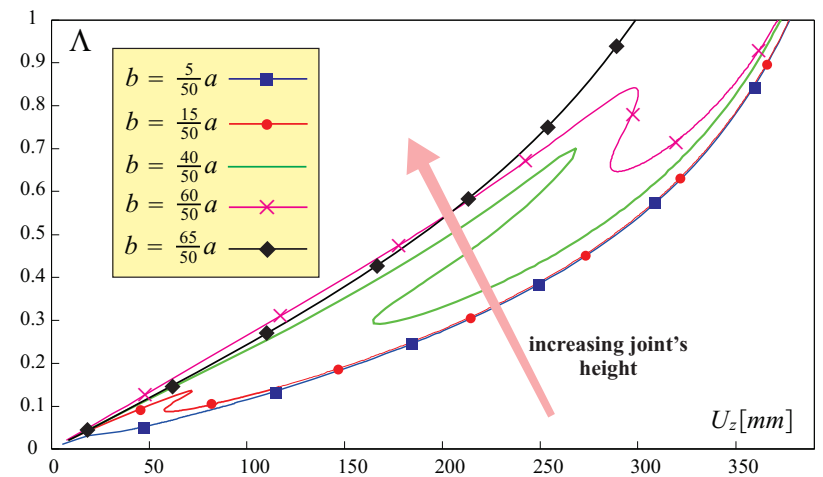

(a) SANISP4 based

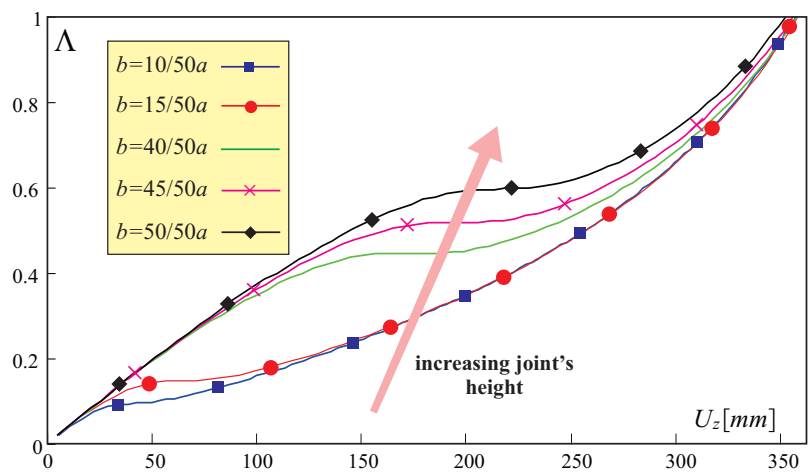

(b) SANISP11 based

Figure 28. Joint's effects for the SANISP4 and SANISP11 based configurations.

\section{Conclusions}

Post-buckling investigations of composite PrandtlPlane Joined Wings, with particular focus on the fundamental physical aspects leading to the instability and nonlinear structural response, have been presented for the first time.

Previous analyses of this innovative configuration focused on linear structural and aeroelastic models. A first attempt towards an accurate simulation and fundamental understanding 
of the critical and post-critical conditions has been presented in Reference [27] where the case of isotropic materials was discussed. The effects of the geometrical parameters (joint's dimensions and sweep angles) showed a very complex nonlinear response which could not be predicted with the standard linear analysis but needs to be properly taken into account even at the very early stages of the design. This is particularly important since the true critical load can often be over predicted when a classical linear eigenvalue approach (linear buckling analysis) is adopted. Reference [27] showed that increasing the joint's height is beneficial. Moreover, it can be identified an interval of joint's heights in which the snapbuckling instability occurs. This was defined as Snap-Buckling Region (SBR). SBR could have a significant relevance in the conceptual design of PrandtlPlane configurations, since a snap-buckling type of instability must be avoided.

Reference [27] also showed that the torsional-bending coupling consequence of the geometric layout (swept wings) can dramatically worsen the stability properties. Finally, it was also presented that the load repartition between the upper and lower wings has a significant effect on the nonlinear response. In particular, for the common PrandtlPlane layout featuring a swept-back lower wing and a swept-forward upper wing, a higher percentage of the load on the upper wing was showed reducing the risks of buckling.

However, several conceptual and theoretical aspects needed to be addressed. This was accomplished in the present work. In particular, the effect of anisotropy was extensively investigated by the adoption of composite materials. The following analyses have been carried out.

\section{Unswept Case with Different Isotropic Materials for the Upper and Lower Wings}

An unswept joined-wing layout was investigated. Different isotropic materials were selected for the upper and lower wings. The lower-to-upper-wing stiffness ratio showed to be the driving parameter as far as snap-buckling instability was concerned. In particular, a counterintuitive result was found: the main mechanism which led to the instability (under nonaerodynamic conservative loads) was not due to the compression. In fact, increasing the stiffness of the upper wing via incrementing the elastic modulus enhances the extensional stiffness (in theory beneficial because the upper wing is compressed), but increases the possibility of having buckling. From the Eulerian compressed column analogy this is a highly unexpected result. The over constrained nature of the Joined Wing (compared to the classical cantilevered wings) and the non-planar geometry are responsible of this complex behavior. 


\section{Unswept Case and Adoption of Orthotropic Materials}

Additional insight on the nature of the snap-buckling was gained by the adoption of a singlelamina orthotropic plate for the upper wing. This choice was useful to detect which stiffness ratio was actually important for the snap-buckling occurrence. It was found that the ratio between the extensional stiffnesses and the ratio between the bending stiffnesses were the primary parameters affecting the Joined Wing's instability properties. As for the isotropic case, it was found that the lower wing's stiffness should be larger than the upper wing's one and the counterintuitive nature of the type of instability was confirmed. However, the single-lamina orthotropic upper wing had the drawback of presenting the lower-to-upperwing extensional stiffness ratio equal to the lower-to-upper-wing bending stiffness ratio: it was not possible to truly separate the relative importance between the extensional and bending effects on the instability.

The problem was addressed by adopting a multilayered orthotropic laminate for the upper wing. This was very useful since for this case the lower-to-upper-wing extensional stiffness ratio is in general different than the lower-to-upper-wing bending stiffness ratio. Several investigations showed then the true driving mechanism to the instability: the snapbuckling occurrence is mainly sensitive to the lower-to-upper-wing bending stiffness ratio. In particular, it was shown that increasing the lower wing's bending stiffness was beneficial, whereas incrementing the upper wing's bending stiffness had a negative effect on the stability of the Joined Wing.

\section{Type of Joint's Connection and its Effects on Snap-Buckling of Unswept Joined Wings}

The bending moment transferring through the joint is one of the leading mechanisms involved in the instability. This was demonstrated by modifying the joint-to-wing connection. In particular, a quasi-hinge type of connection was implemented by inserting a hinge at the joint-to-upper-wing or joint-to-lower-wing junction and a torsional spring opposing the relative rotation between the joint and the upper/lower wing. A small value of the spring stiffness was selected and the bending moment transferred to the upper wing was reduced compared to the fixed type of joint previously investigated. On a pure stability perspective, this provided a great benefit because the snap-buckling was eventually eliminated, thus, confirming the relevant importance of the bending moment transferring in the snap occurrence. As a drawback, however, the configurations with the quasi-hinge connection showed a less stiff response. Moreover, when the quasi-hinge was positioned between the joint and upper wing a softening tendency was observed in respect of the response of the configuration in which the quasi-hinge connection was established between the joint and the lower wing. 


\section{Unswept Case and Adoption of Anisotropic Materials (Composites)}

While the lower wing was assumed to be isotropic, the upper wing was simulated first with a multilayered orthotropic laminate and second with a multilayered anisotropic laminate presenting identical lower-to-upper-wing bending stiffness ratio. The orthotropic case experienced a strong snap-type instability, whereas the anisotropic case did not have the instability at all in the range of explored load levels and presented a stiffening effect. Considering the fact that the geometry (unswept Joined Wings) does not introduce any bending-torsion coupling whereas the anisotropic laminate does, it was deduced that the material-induced bending-torsion coupling is important in the nonlinear response.

The bending-torsion coupling was further investigated by selecting a single-lamina with fibers generically oriented. The lower-to-upper-wing bending stiffness ratio and the primary bending-torsional deformation coupling term $D_{26}$ was also monitored. The investigation showed that in some cases the bending-torsional coupling is beneficial and in other cases it deteriorates the stability properties of the Joined Wing. A further insight of this coupling and its effects on the instability could be gained from the observation of the joint's rotation with respect to the wing-span direction. This rotation is heavily affected by the torsionalbending coupling. In particular, a properly designed laminate could increase or decrease the rotation of the joint with enhanced or worsened stability properties.

\section{Swept Case with Different Isotropic Materials for the Upper and Lower Wings}

The swept Joined Wing presents a torsional-bending coupling even if the same isotropic material is adopted for both the upper and lower wings. To gain further insight, the upper wing was selected to be isotropic but with different elastic modulus compared to the lower wing. Several investigations showed that to avoid snap-buckling a substantially larger lowerto-upper-wing bending stiffness ratio was necessary. A direct observation of the deformed shapes confirmed that when this type of instability occurs the rotation (with respect to the wing span axis) of the joint is significant.

\section{Swept Case and Torsional Effects}

The relative importance of torsional effects was further investigated selecting the reference swept joined-wing configuration, and changing the torsional stiffness. This was easily accomplished by adopting an orthotropic material and changing the shear modulus. When the torsional stiffness was increased, the response showed a tendency to have an abrupt snapbuckling, typical of the unswept case. This should confirm that, limiting the torsional effects introduced by the geometry, the configuration tends to behave as in the unswept case. 


\section{Swept Case and Anisotropy Effects}

A physical insight on the response of PrandtlPlane Joined Wings was gained by the introduction of coupling effects associated with the anisotropy of the material. While the lower wing was maintained isotropic, the upper wing was simulated with a single-ply generically oriented material. The analyses showed a very complex bending-torsion interaction: even for a relatively simple single-ply upper wing there could be identified two distinct and unsymmetrically located regions in which the snap-buckling occurs. In other words, the Snap Buckling Region (i.e., range of fiber orientation's angles for which the system undergoes a snap-buckling instability) is difficult to predict from pure intuitive considerations.

\section{Joint's Height Effects in the Case of Anisotropic Swept Joined Wings}

For the isotropic case examined in Reference [27] the joint's height was varied and it was found that an increase of the height was beneficial. A similar investigation has been carried out in this work with the adoption of composite materials. It was found out that the joint's height is beneficial even when the composite materials are used.

The investigations presented in this work shed some light on the very complex nonlinear response of Joined Wings. Results showed that the snap-buckling load is quite sensitive to the bending moment transferred by the joint to the upper wing. Thus, both the type of joint's connection and bending stiffness of the wings play a significant role.

When the bending-torsion coupling is introduced because of either the geometry (sweep angles) or the material (composites) or both, predicting a priori if the above mentioned effects are beneficial or not is an extremely challenging task. The simulations conducted in this work seem to suggest that the rotation of the joint about the wing span axis is an important factor for further gaining insight on instability and its properties.

Future works focusing on nonlinear effects of Joined Wings will discuss the theoretical challenges and physical implications when mechanical non-conservative loadings of a follower type and aerodynamic forces are taken into account. Specifically, in Reference [36] a Vortex Lattice Method has been used for this purpose. A further area needing some studies is the characterization of stability properties under a dynamical systems perspective ${ }^{34}$.

\section{Acknowledgements}

The authors acknowledges the support by San Diego State University (College of Engineering). 


\section{References}

${ }^{1}$ Letcher, J., "V-Wings and Diamond-Ring Wings of Minimum Induced Drag," Journal of Aircraft, Vol. 8, 1972, pp. 605-607.

${ }^{2}$ Wolkovitch, J., "The Joined Wing Aircraft: an Overview," Journal of Aircraft, Vol. 23, 1986.

${ }^{3}$ Lange, R. H., Cahill, J. F., Bradley, E. S., Eudaily, R. R., Jenness, C. M., and Macwilkinson, D. G., "Feasibility Study of the Transonic Biplane Concept for Transport Aircraft Applications," 1974, NASA CR-132462, Lockheed-Georgia Company.

${ }^{4}$ Miranda, L. R., "Boxplane Wing and Aircraft," US Patent 3,834,654, September 1974.

${ }^{5}$ Frediani, A., "Large Dimension Aircraft," US Patent 5,899,409, 1999.

${ }^{6}$ Frediani, A., "New Large Aircraft," European Patent EP 0716978B1, 20 March 2002.

${ }^{7}$ Livne, E., "Future of Airplane Aeroelasticity," Journal of Aircraft, Vol. 40, 2003, pp. 1066-1092.

${ }^{8}$ Livne, E. and Weisshaar, T. A., "Aeroelasticity of Nonconventional Airplane Configurations-Past and Future," Journal of Aircraft, Vol. 40, 2003, pp. 1047-1065.

${ }^{9}$ Prandtl, L., "Induced Drag of Multiplanes," Tech. Rep. TN 182, NACA, March 1924.

${ }^{10}$ Scott, M., Enke, A., and Flanagan, J., "SensorCraft Free-Flying Aeroservoelastic Model Design and Fabrication," Presented at the 52 ${ }^{\text {nd }}$ AIAA/ASME/ASCE/AHS/ASC Structures, Structural Dynamics \& Materials Conference, Denver, Colorado, 4-7 April 2011.

${ }^{11}$ Buttazzo, G. and Frediani, A., editors, Variational Analysis and Aerospace Engineering: Mathematical Challenges for Aerospace Design Contributions from a Workshop held at the School of Mathematics in Erice, Italy, Springer US, 2012.

${ }^{12}$ Gern, F. H., Ko, A., Sulaeman, E., Gundlach, J., Kapania, R. H., and Haftka, R. T., "Multidisciplinary Design Optimization of a Transonic Commerical Transport with Strut-Braced Wing," Journal of Aircraft, Vol. 38, 2001, pp. 1006-1014.

${ }^{13}$ Gur, O., Bhatia, M., Schetz, J. A., Mason, W. H., Kapania, R. K., and Mavris, D. N., "Design Optimization of a Truss-Braced-Wing Transonic Transport Aircraft," Journal of Aircraft, Vol. 47, No. 6, 2010, pp. 1907-1917.

${ }^{14}$ Reichenbach, E., Castelluccio, M., and B., S., "Joined Wing Sensorcraft Aeroservoelastic Wind Tunnel Test Program," Presented at the 52 ${ }^{\text {nd }}$ AIAA/ASME/ASCE/AHS/ASC Structures, Structural Dynamics \& Materials Conference, Denver, Colorado, 4-7 April 2011.

${ }^{15}$ Boston, J., Swenson, E., and Kunz, D., "Experimens with Geometric Non-Linear Coupling for Analytical Validation," Presented at the $51^{\text {st }}$ AIAA/ASME/ASCE/AHS/ASC Structures, Structural Dynamics \& Materials Conference, Orlando, Florida, 12-15 April 2010.

${ }^{16}$ Paletta, N., Belardo, M., and Pecora, M., "Load Alleviation on a Joined-Wing Unmanned Aicraft," Journal of Aircraft, Vol. 47, 2010, pp. 2005-2016.

${ }^{17}$ Sotoudeh, Z. and Hodges, D. H., "Incremental Method for Structural Analysis of Joined-Wing Aircraft," Journal of Aircraft, Vol. 48, 2011, pp. 1588-1601.

${ }^{18}$ Kim, Y. I., Park, G. J., Kolonay, R. M., Blair, M., and Canfield, R. A., "Nonlinear Response Structural Optimization of a Joined Wing Using Equivalent Loads," AIAA Journal, Vol. 46, 2008.

${ }^{19}$ Chambers, J. R., Innovation in Flight: Research of the NASA Langley Research Center on Revolutionary Advanced Concepts for Aeronautics, No. 39 in Monograph in Aerospace History, NASA, November 2005, NASA SP 2005-4539. 
${ }^{20}$ Weisshaar, T. A. and Lee, D. H., "Aeroelastic Tailoring of Joined-Wing Configurations," 43nd AIAA/ASME/ASCE/AHS/ASC Structures, Structural Dynamics and Materials Conference, Denver, CO,, 22-25 April 2002.

${ }^{21}$ Demasi, L. and Livne, E., "Contributions to Joined-Wing Aeroelasticity," Presented at the International Forum on Aeroelasticity and Structural Dynamics Conference, Seattle, Washington, 21-25 June 2009 .

${ }^{22}$ Demasi, L. and Livne, E., "Exploratory Studies of Joined Wing Aeroelasticity," Presented at the 46th AIAA/ASME/ASCE/AHS/ASC Structures, Structural Dynamics \& Materials Conference, Austin, Texas, 18-21 April 2005.

${ }^{23}$ Demasi, L. and Livne, E., "The Structural Order Reduction Challenge in the Case of Geometrically Nonlinear Joined-Wing Configurations," Presented at the 48th AIAA/ASME/ASCE/AHS/ASC Structures, Structural Dynamics \& Materials Conference, Honolulu, Hawaii, 23-26 April 2007.

${ }^{24}$ Tiso, P. and Jansen, E., "A Finite Element Based Reduction Method for Nonlinear Dynamics of Structures," Presented at the $46^{\text {th }}$ AIAA/ASME/ASCE/AHS/ASC Structures, Structural Dynamics \& Materials Conference, Austin, Texas, 18-21 April 2005.

${ }^{25}$ Rixen, D. J. and Tiso, P., "Reduction Methods for Mems Nonlinear Dynamic Analysis," Presented at the IMAC-XXVIII International Modal Analysis Conference, 1-4 February 2010, Jacksonville, Florida.

${ }^{26}$ Demasi, L. and Palacios, A., "A Reduced Order Nonlinear Aeroelastic Analysis of Joined Wings Based on the Proper Orthogonal Decomposition," Presented at the $51^{\text {st }}$ AIAA/ASME/ASCE/AHS/ASC Structures, Structural Dynamics \& Materials Conference, Orlando, Florida, 12-15 April 2010.

${ }^{27}$ Demasi, L., Cavallaro, R., and Razon, A., "Post-critical Analysis of PrandtlPlane Joined-Wing Configurations," AIAA Journal, Vol. 51, No. 1, 2013, pp. 161-177.

${ }^{28}$ Giles, L. G., "Equivalent Plate Modeling for Conceptual Design of Aircraft Wing Structures," Tech. Rep. NASA-TM-111263, NASA, Septemeber 1995.

${ }^{29}$ Stearman, R., Lin, H.-H., and Jhou, J., "Influence of Joint Fixity on the Aeroelastic Characteristics of a Joined Wing Structure," No. AIAA-90-0980-CP, AIAA/ASME/ASCE/AHS/ASC Structures, Structural Dynamics and Materials Conference, 31st, Long Beach, CA, American Institute of Aeronautics and Astronautics, Washington, DC, April 2-4 1990, pp. 1442-1454.

${ }^{30}$ Levy, R. and Gal, E., "Triangular Shell Element for Large Rotations Analysis," AIAA Journal, Vol. 41, 2003, pp. 2505-2508.

${ }^{31}$ Crisfield, M., Non Linear Finite Element Analysis of Solid and Structures, Vol. 1, John Wiley \& Sons, 1991.

${ }^{32}$ Cavallaro, R., Demasi, L., and Passariello, A., "Nonlinear Analysis of PrandtlPlane Joined Wings - Part II: Effects of Anisotropy," No. AIAA 2012-1462, 53rd AIAA/ASME/ASCE/AHS/ASC Structures, Structural Dynamics, and Materials Conference, Honolulu, Hawaii, 23-26 April 2012.

${ }^{33}$ Bisplinghoff, R. and Ashley, H., Principles of Aeroelasticity, Dover Phoenix Editions, Dover Publications, 2002.

${ }^{34}$ Cavallaro, R., Demasi, L., and Bertuccelli, F., "Risks of Linear Design of Joined Wings: a Nonlinear Dynamic Perspective in the Presence of Follower Forces," No. AIAA 2013-1558, 54rd AIAA/ASME/ASCE/AHS/ASC Structures, Structural Dynamics, and Materials Conference, Boston, Massachusetts, American Institute of Aeronautics and Astronautics, 8-11 April 2013.

${ }^{35}$ Blair, M., Canfield, R. A., and Roberts Jr., R. W., "Joined-Wing Aeroelastic Design with Geometric Nonlinearity," Journal of Aircraft, Vol. 42, 2005. 
${ }^{36}$ Demasi, L., Cavallaro, R., and Bertuccelli, F., "Post-Critical Analysis of Joined Wings: the Concept of Snap-Divergence as a Characterization of the Instability," No. AIAA 2013-1559, 54rd AIAA/ASME/ASCE/AHS/ASC Structures, Structural Dynamics, and Materials Conference, Boston, Massachusetts, American Institute of Aeronautics and Astronautics, 8-11 April 2013. 